\title{
Cell Stem Cell
}

\section{Repression of endogenous retroviruses prevents antiviral immune response and is required for mammary gland development}

\section{Graphical abstract}

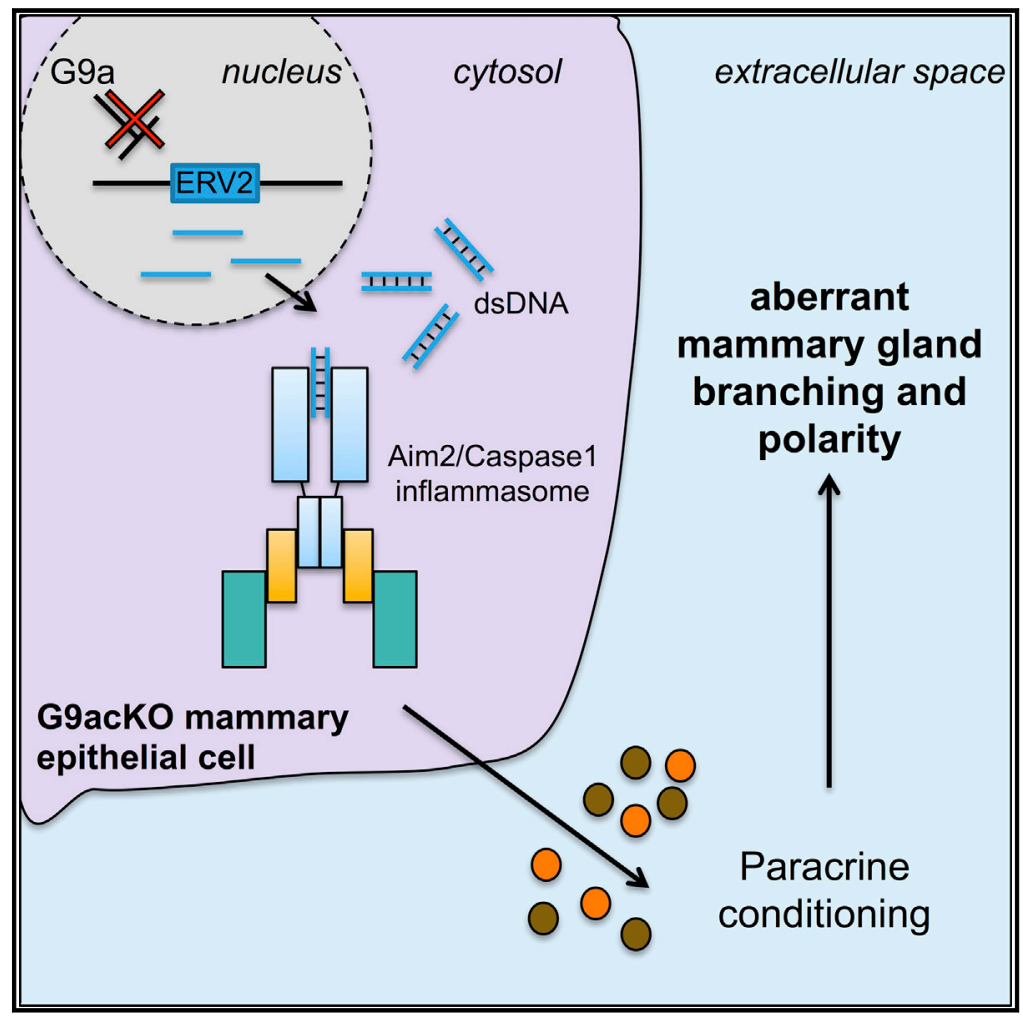

\section{Highlights}

- Impaired mammary gland development upon loss of H3K9 methyltransferase G9a

- G9a loss opens chromatin in regions harboring endogenous retroviruses

- Expression of endogenous retroviruses triggers cellular antiviral response

- Inhibition of cell's antiviral response ameliorates the observed mammary gland defects

\section{Authors}

Alexandra Avgustinova,

Carmelo Laudanna, Mónica Pascual-García, ..., Holger Heyn, Juan M. Vaquerizas, Salvador Aznar Benitah

\section{Correspondence}

aavgustinova@fsjd.org (A.A.), salvador.aznar-benitah@ irbbarcelona.org (S.A.B.)

\section{In brief}

The role of chromatin accessibility in tissue development is not well understood. Avgustinova and colleagues demonstrate that increased chromatin accessibility results in delayed and aberrant mammary gland development. These defects are partially cell autonomous and in part caused by an altered mammary milieu following transcriptional derepression of endogenous retroviruses. 


\title{
Cell Stem Cell
}

\section{Repression of endogenous retroviruses prevents antiviral immune response and is required for mammary gland development}

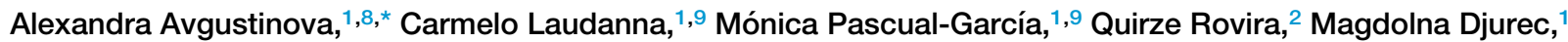 \\ Andres Castellanos, ${ }^{1}$ Uxue Urdiroz-Urricelqui, ${ }^{1}$ Domenica Marchese, ${ }^{3}$ Neus Prats, ${ }^{1}$ Alexandra Van Keymeulen, ${ }^{4}$ \\ Holger Heyn, ${ }^{3,5}$ Juan M. Vaquerizas, ${ }^{2,6}$ and Salvador Aznar Benitah ${ }^{1,7,10, *}$ \\ ${ }^{1}$ Institute for Research in Biomedicine (IRB Barcelona), The Barcelona Institute of Science and Technology (BIST), Barcelona, Spain \\ ${ }^{2}$ Max Planck Institute for Molecular Biomedicine, Münster, Germany \\ ${ }^{3}$ CNAG-CRG, Center for Genomic Regulation (CRG), The Barcelona Institute of Science and Technology (BIST), Barcelona, Spain \\ ${ }^{4}$ Laboratory of Stem Cells and Cancer, Université Libre de Bruxelles (ULB), Brussels, Belgium \\ 5Universitat Pompeu Fabra (UPF), Barcelona, Spain \\ ${ }^{6} \mathrm{MRC}$ London Institute of Medical Sciences, Institute of Clinical Sciences, Faculty of Medicine, Imperial College London, Du Cane Road, \\ London W12 ONN, UK \\ ${ }^{7}$ Catalan Institution for Research and Advanced Studies (ICREA), Barcelona, Spain \\ ${ }^{8}$ Present address: Institut de Recerca Sant Joan de Déu, Esplugues de Llobregat, Spain \\ 9These authors contributed equally \\ 10Lead contact \\ *Correspondence: aavgustinova@fsjd.org (A.A.), salvador.aznar-benitah@irbbarcelona.org (S.A.B.) \\ https://doi.org/10.1016/j.stem.2021.04.030
}

\section{SUMMARY}

The role of heterochromatin in cell fate specification during development is unclear. We demonstrate that loss of the lysine 9 of histone H3 (H3K9) methyltransferase G9a in the mammary epithelium results in de novo chromatin opening, aberrant formation of the mammary ductal tree, impaired stem cell potential, disrupted intraductal polarity, and loss of tissue function. G9a loss derepresses long terminal repeat (LTR) retroviral sequences (predominantly the ERVK family). Transcriptionally activated endogenous retroviruses generate double-stranded DNA (dsDNA) that triggers an antiviral innate immune response, and knockdown of the cytosolic dsDNA sensor Aim2 in G9a knockout (G9acKO) mammary epithelium rescues mammary ductal invasion. Mammary stem cell transplantation into immunocompromised or G9acKO-conditioned hosts shows partial dependence of the G9acKO mammary morphological defects on the inflammatory milieu of the host mammary fat pad. Thus, altering the chromatin accessibility of retroviral elements disrupts mammary gland development and stem cell activity through both cell-autonomous and non-autonomous mechanisms.

\section{INTRODUCTION}

Modulation of chromatin accessibility in non-coding regions through epigenetic regulation might be important for correct cell fate specification during embryonic development, but it is unclear whether this is critical for proper tissue development and maintenance. We recently showed that keratin 14 (K14)specific deletion of the histone methyltransferase G9a leads to severe reduction of dimethylated lysine 9 of histone $\mathrm{H} 3$ (H3K9me2) levels, concomitant with significant de novo chromatin opening in the epidermis (Avgustinova et al., 2018). H3K9me2 generates low chromatin accessibility, contributing to gene repression at euchromatin and formation of heterochromatin (Tachibana et al., 2005). Despite increased chromatin opening following epidermal deletion of G9a, very few proteincoding genes show changes in their expression, and epidermal development, morphology, and cell fate specification remain largely unscathed. However, upon cellular stress (in the form of DNA damage), epidermal stem cells lacking G9a are more susceptible to malignant transformation (Avgustinova et al., 2018). Epigenetic mechanisms are largely dispensable for maintenance of adult stem cell identity but mediate the stem cell response to tissue-specific stress (Avgustinova and Benitah, 2016). We thus wanted to find out whether increased chromatin accessibility following G9a deletion might have different outcomes in other tissues.

Here we investigated how chromatin opening following G9a ablation affects mammary gland development and cell fate specification. The mouse mammary gland develops largely postnatally, making it an excellent model system for studying tissue development and differentiation. At birth, the mammary epithelium consists of a rudimentary ductal tree that undergoes rapid 

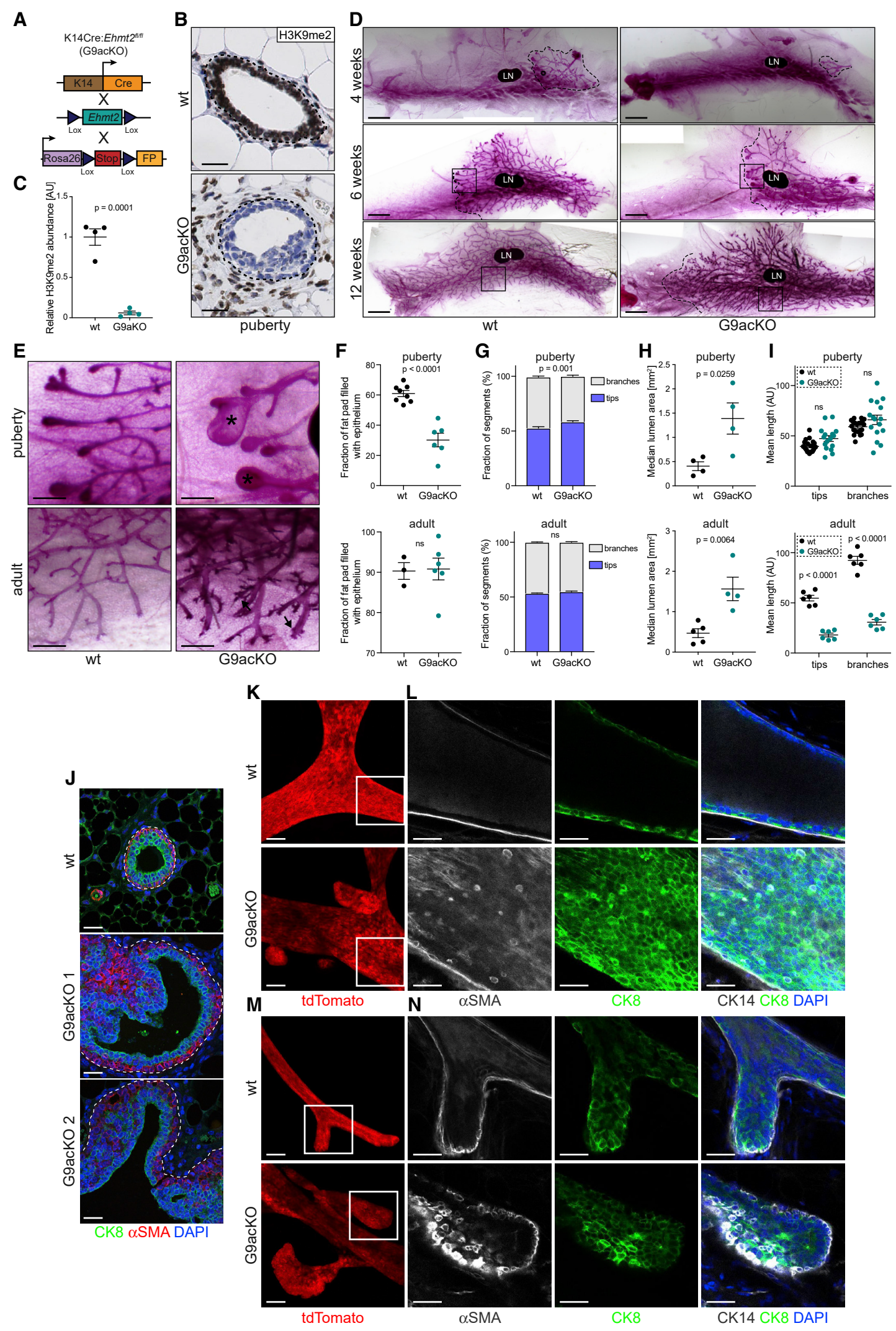


\section{Cell Stem Cell} Article

expansion in response to hormonal signals during puberty until the homeostatic adult state is reached (Visvader and Stingl, 2014). During adulthood, the mammary epithelium remains plastic and is sensitive to hormonal cues, facilitating lobulo-alveolar differentiation during pregnancy and involution after weaning. We demonstrate that G9a loss results in severely disrupted development of the mammary epithelium, impaired stem cell potential, and profound alterations in epithelial polarity and extracellular matrix deposition. Intriguingly, despite the striking disruption of development in G9a-ablated mammary epithelia, lineage specification and fidelity remained largely unperturbed. However, mammary epithelial cells lacking G9a aberrantly expressed long terminal repeat (LTR) endogenous retroviruses, which can activate an antiviral innate immune response. Blocking the cellular response to LTR endogenous retroviral derepression ameliorates morphological defects in mammary epithelia lacking G9a. Finally, we provide evidence that suggests that cell-autonomous and non-autonomous mechanisms contributed to the observed morphological abnormalities in G9a knockout (G9acKO) mammary glands.

\section{RESULTS}

G9a loss delays mammary gland development

Mice carrying a K14-specific G9a knockout (G9acKO; Figure 1A) exhibited severely reduced levels of $\mathrm{G} 9 \mathrm{a}$ and $\mathrm{H} 3 \mathrm{~K} 9 \mathrm{me} 2$ in luminal and basal cells of the mammary epithelium (Figures 1B, $1 \mathrm{C}$, and S1A-S1C). Although luminal cells do not express cytokeratin 14, loss of G9a and $\mathrm{H} 3 \mathrm{~K} 9 \mathrm{me} 2$ in this lineage is expected because both lineages originate from a common K14-positive progenitor cell during embryonic development (Jonkers et al., 2001; Sun et al., 2010).

Ductal invasion into the mammary fat pad was delayed severely in early-pubertal (4-week-old) and pubertal (6-weekold) G9acKO mice (Figure 1D), although most G9acKO mammary glands filled the entire mammary fat pad in 12-week-old (adult) mice. Morphologically, the 6-week-old (pubertal) G9acKO mammary tree was characterized by severely dis- tended and abnormal terminal end buds (TEBs) and a slight increase in the proportion of tip versus branch segments (consistent with an overall smaller mammary tree) (Figures 1E-1G). Pubertal and adult G9acKO mammary epithelia displayed distended ducts (Figure $1 \mathrm{H}$ ), whereas adult G9acKO mammary epithelia were more branched, with a large number of abortive ductal tips and overall shorter tip and ductal branch segments compared with wild-type (WT) littermate controls (Figure 1I).

\section{Aberrant cell polarity and extracellular matrix in G9acKO mammary epithelium}

The bilayered mammary epithelium comprises two principal lineages: (1) luminal cells, which line the ductal lumen and are responsible for hormone sensing and milk production during pregnancy, and (2) basal cells, which envelop the luminal layer, directly contact the stroma and the basement membrane, and provide the contractile force for milk ejection during lactation. WT mammary ducts had correct expression of both layers of luminal cells (expressing cytokeratin 8 [CK8]) and basal cells (expressing $\alpha$-smooth muscle actin [ $\alpha \mathrm{SMA}])$; in contrast, G9acKO mammary epithelium had intermingled luminal and basal cells (as shown by $\alpha$ SMA and CK8; in both ducts and TEBs in pubertal mice and mainly at the tip segments in adult mice), and basal cells had a columnar rather than a spindle shape, reminiscent of luminal cells (Figures $1 \mathrm{~J}-1 \mathrm{~N}$ and S1D-S1G).

In G9acKO mammary glands, neither the tight-junction protein zona occludens 1 (ZO-1) nor the apical membrane domain marker p-ezrin (Thr567)/p-radixin (Thr564)/p-moesin (Thr558) (pERM) (Godde et al., 2014) were limited to the apical surface of the luminal epithelial cells, as would be expected (Ewald et al., 2008), indicating loss of apico-basal polarity (Figures 2A, S2A, and S2B). Strikingly, ZO-1 also marked the baso-lateral surface of a subset of luminal cells, identified additional rudimentary lumina within the multilayered G9acKO mammary epithelium, and showed nuclear localization in adult G9acKO epithelia, consistent with immature cell-cell contacts (Gottardi et al., 1996; Figures 2A and S2A). Furthermore, E-cadherin and pERM were localized aberrantly on the apical surface of G9acKO basal epithelial cells (Figure S2B).

Figure 1. G9a loss leads to delayed mammary gland development and mammary lineage intermingling

(A) Schematic of G9acKO mouse line generation. Ehmt2, the gene encoding G9a; FP, fluorescent protein, eYFP or tdTomato, as indicated.

$(B$ and C) Immunohistochemistry (B) and quantification (C) of H3K9me2 in mammary fat pads from 6-week-old (pubertal) WT or G9acKO mice. $n=4$ mice per group. In (B), nuclei were counterstained with hematoxylin. The dashed line delineates the epithelium/stroma boundary. Scale bar, $25 \mu \mathrm{m}$. In (C), data shown are mean \pm SEM of independent animals. Two-sided Student's t test.

(D) Carmine-alum staining of mammary wholemounts from WT or G9acKO mice (4, 6, or 12 weeks old) (stitched images; STAR Methods). The dashed line indicates the mammary tree invading front. LN, lymph node. Images are representative. 4-week-old mice: $n=3 ; 6$-week-old mice: $n=8(W T), n=6(G 9 a c K O)$; adult mice: $n=3(W T), n=6$ (G9acKO). Scale bar, $2 \mathrm{~mm}$.

(E) Magnification of areas highlighted in (D). Asterisks indicate distended TEBs of G9acKO mammary ducts. Scale bar, $0.5 \mathrm{~mm}$.

(F-I) Quantification of mammary epithelium in pubertal or adult mice (WT or G9acKO) for areas in mammary fat pads filled by epithelium (F), fraction of branch versus tip segments $(\mathrm{G})$, median lumen area $(\mathrm{H})$, and mean branch and tip segment length $(\mathrm{I})$. Data are mean \pm SEM. In $(\mathrm{F})$, each data point represents an independent animal. Pubertal mice: $n=8$ (WT), $n=6$ (G9acKO); adult mice: $n=3(W T), n=6$ (G9acKO) mice. Two-sided Student's t test. In (G), pubertal mice: $n=7$ (WT), $n=6$ (G9acKO); adult mice: $n=3$. Two-way ANOVA with Dunnett multiple comparisons test. In $(H)$, each data point represents an independent animal. $n=4$ mice for each group. Two-sided Student's $t$ test. In (l), each data point represents a region of interest (ROI) (with 3 ROIs per animal). Pubertal mice: $n=7($ WT), $n=6$ (G9acKO); adult mice: $\mathrm{n}=3$. Two-way ANOVA with Dunnett multiple comparisons test.

(J) Immunofluorescence for $\alpha$ SMA (red) and CK8 (green) on mammary fat pads from pubertal WT or G9acKO mice. $\mathrm{n}=4$ mice for each group. Nuclei were counterstained with DAPI (blue). Two examples are shown for G9acKO to capture phenotypic heterogeneity. The dashed line delineates the epithelium/stroma boundary. Scale bar, $25 \mu \mathrm{m}$.

(K-N) Endogenous Rosa26tdTomato signal (red, K and M) or immunofluorescence staining (L and N) for CK8 (green) and $\alpha \mathrm{SMA}$ (gray) on mammary wholemounts from pubertal WT or G9acKO mice. Nuclei were counterstained with DAPI (blue). G9a loss leads to cellular intermingling and basal cell multilayering in both ductal segments ( $K$ and $L)$ and TEBs ( $M$ and $N)$. Scale bars, $50 \mu \mathrm{m}(K$ and $M)$ and $25 \mu \mathrm{m}(\mathrm{L}$ and $\mathrm{N})$.

See also Figure S1. 


\section{$\boldsymbol{\infty}$ CellPress}

A
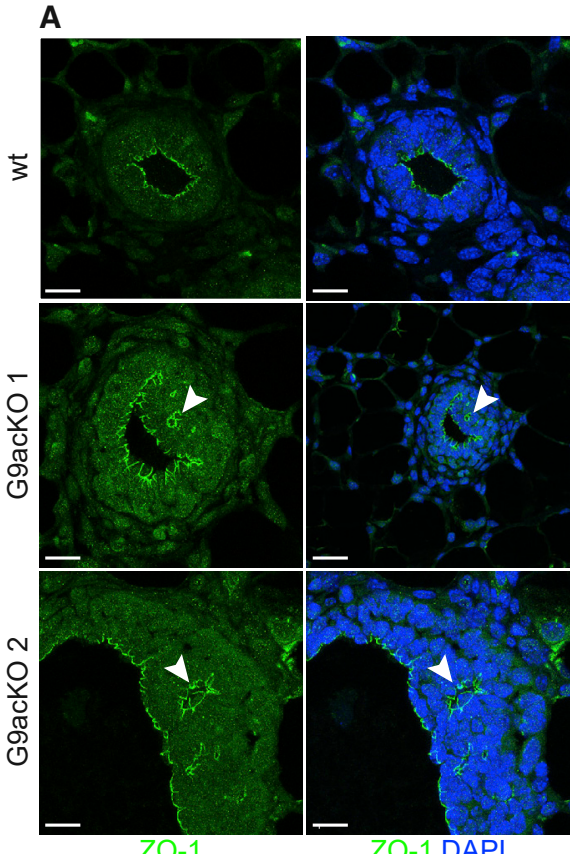

B

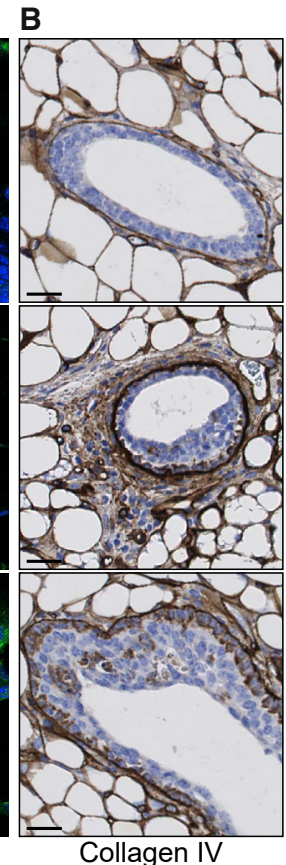

C
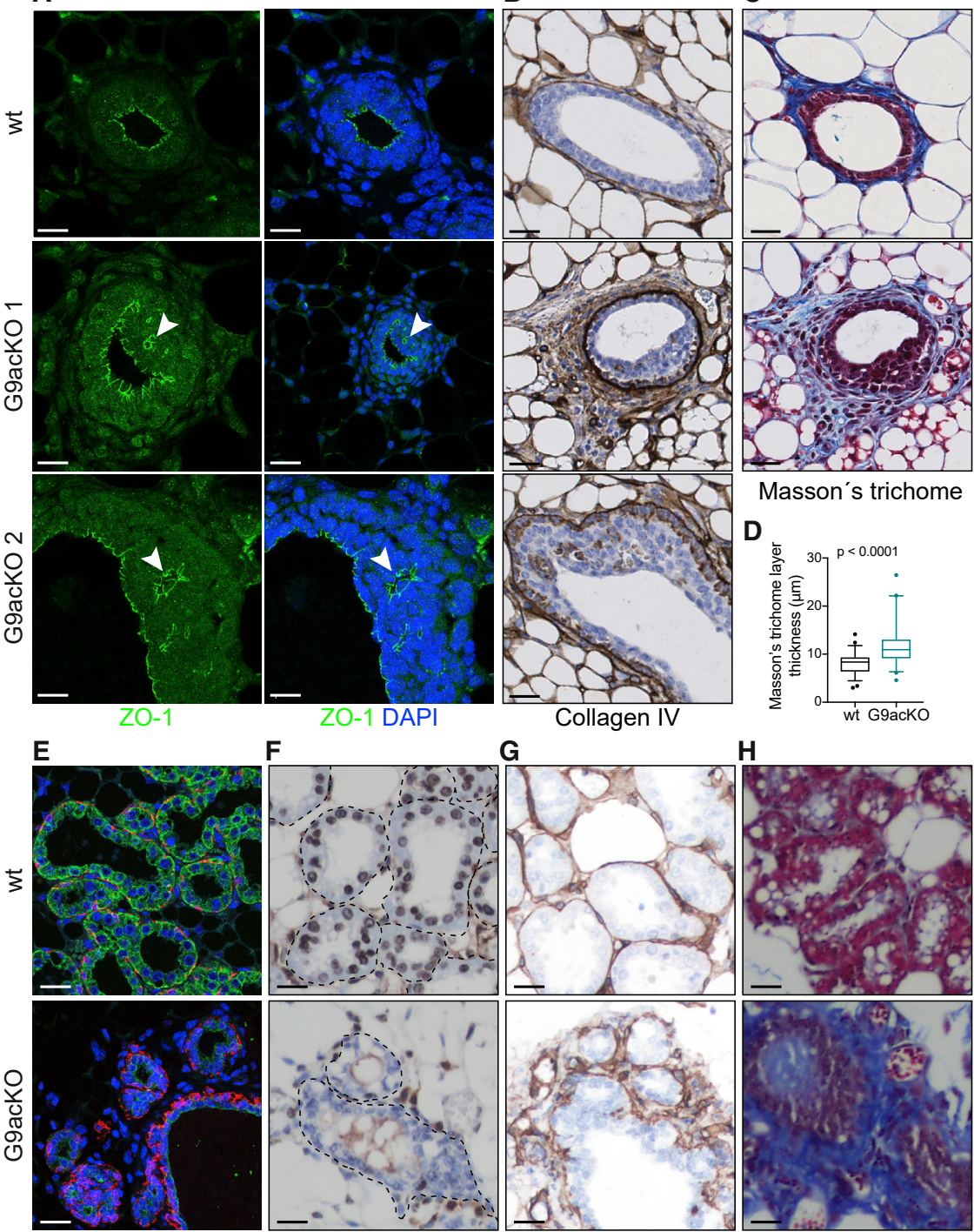

CK8 aSMA DAPI
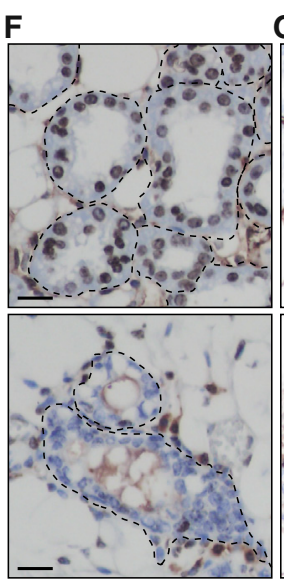

H3K9me2
G

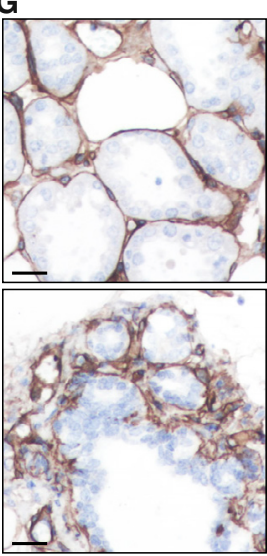

Collagen IV
$\mathrm{H}$

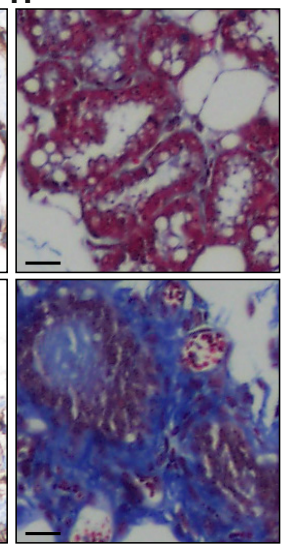

Masson's trichome

Figure 2. G9a loss disrupts tissue polarity and function

(A-C) Immunofluorescence studies of mammary fat pads from pubertal WT or G9acKO mice, staining (A) ZO-1 (green); nuclei are counterstained with DAPI (blue). Arrowheads indicate aberrant lumina; (B) collagen IV or (C) Masson's trichome staining. For (A) and (B), two examples are shown for G9acKO to capture phenotypic heterogeneity. For $(B)$ and $(C)$, nuclei were counterstained with hematoxylin. $n=4$ mice for each group. Scale bar, $25 \mu \mathrm{m}$. (D) Quantification of the thickness of Masson's trichome layer around mammary ducts in mammary fat pads from pubertal WT or G9acKO mice. Each data point represents an individual duct. WT, $\mathrm{n}=56$ ducts from 4 mice; G9acKO, $\mathrm{n}=47$ ducts from 5 mice. Two-sided Student's t test.

(E-H) Immunofluorescence analyses of mammary fat pads on lactation day 1 from WT or G9acKO mice, staining for (E) $\alpha$ SMA (red) and CK8 (green), with nuclei counterstained with DAPI (blue). A lack of alveolar differentiation in G9acKO mammary epithelia is evident. Shown in $(F)$ is H3K9me2; the dashed line delineates the epithelium/stroma boundary. Shown in $(G)$ are collagen IV and $(H)$ Masson's trichome staining. For $(\mathrm{F})-(\mathrm{H})$, nuclei were counterstained with hematoxylin. $n=3$ mice per group. Scale bars, $25 \mu \mathrm{m}$.

See also Figure S2.

tion and aberrant collagen deposition was present in mid-pregnancy (Figures S2I-S2L), predicting failure of G9acKO mammary tissue function.

\section{Severely reduced stem cell potential of G9acKO mammary epithelium}

We next used the Rosa26YFP-reporter cassette in our transgenic mouse line (Figure 1A) to distinguish the mammary epithelium from stroma by flow cytometry by following YFP-expressing cells

Finally, collagen IV, one of the main constituents of the mammary basement membrane, was more abundant and no longer confined to the epithelial/stromal interface of basal cells in G9acKO mammary glands (Figures 2B and S2C). Indeed, collagen deposition (as measured by Masson's trichome staining) was increased significantly around G9acKO mammary ducts (Figures 2C, 2D, S2D, and S2E). Of note, estrogen receptor alpha $(E R \alpha)$-positive and $E R \alpha$-negative luminal lineages were specified correctly in G9acKO mammary epithelia, albeit with a slight decrease in ER $\alpha$-positive mature luminal cells (Figures S2F and S2G).

Postpartum, tissue expansion and alveologenesis were incomplete in G9acKO mothers on day 1 of lactation (Figures $2 \mathrm{E}$ and $\mathrm{S} 2 \mathrm{H})$, concomitant with a strong decrease in H3K9me2 levels (Figure 2F) and increased collagen deposition (Figures $2 \mathrm{G}$ and $2 \mathrm{H}$ ). G9acKO mothers failed to produce milk, and their pups starved on postnatal day 1 when not cross-fostered to WT mothers (data not shown). The delay in alveolar differentia-
(Figure S3A). Compared with WT mice, pubertal G9acKO mice had a significantly lower proportion of YFP-positive mammary epithelial cells within their mammary fat pads; however, the YFP-positive proportion was recovered in adult G9acKO fat pads, underscoring a developmental delay rather than failure (Figures 3A, 3B, and S3B). Surprisingly, there were no differences in the relative percentage of luminal and basal cells at either developmental time point (Figure 3C).

Assessing the stem cell potential of cells sorted by fluorescence-activated cell sorting (FACS), we observed that colonyforming potential was severely compromised (with reduced colony numbers and size) in luminal and basal G9acKO cells plated in 2D or 3D (Figures 3D-3G and S3C-S3H). However, the gold standard for assessing mammary stem cell potential is epithelial cell transplantation into a cleared mammary fat pad (Shackleton et al., 2006). Because mammary basal cells in G9acKO mice had an altered morphology (and, thus, perhaps distinct expression of cell-specific markers, biasing FACS isolation), we chose to 
A

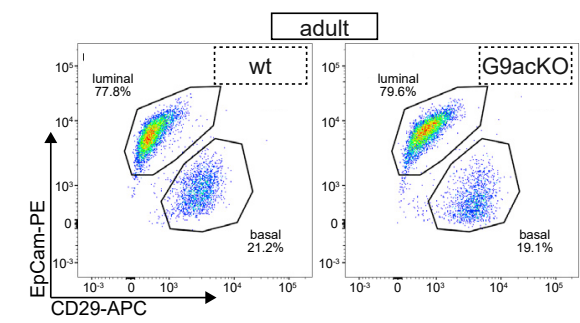

D
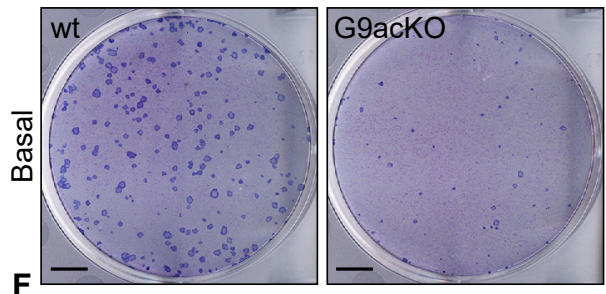

$\mathbf{F}$
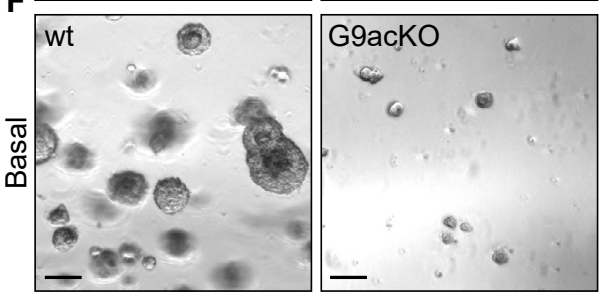

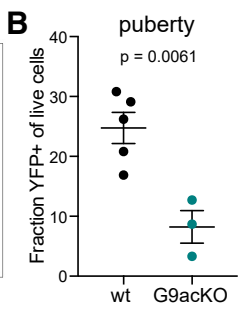

E

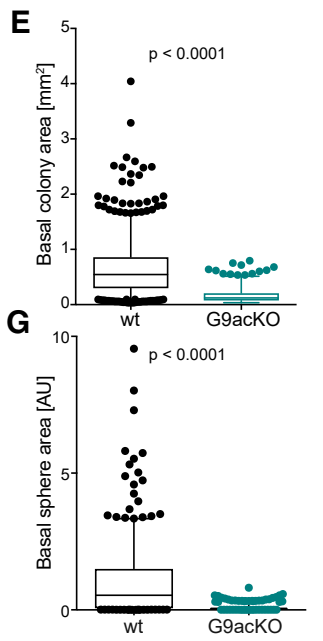

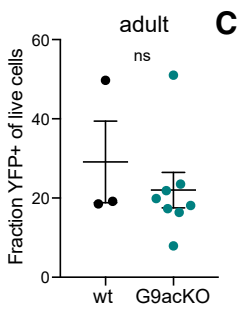

H
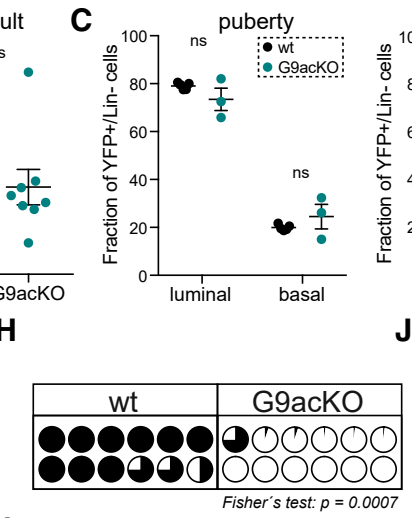

I

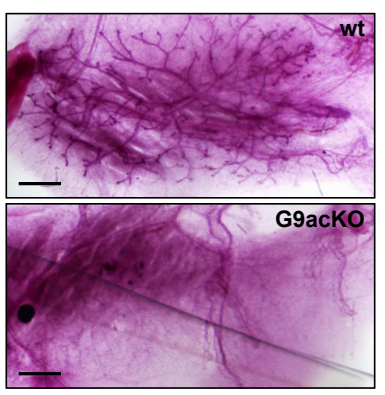

J
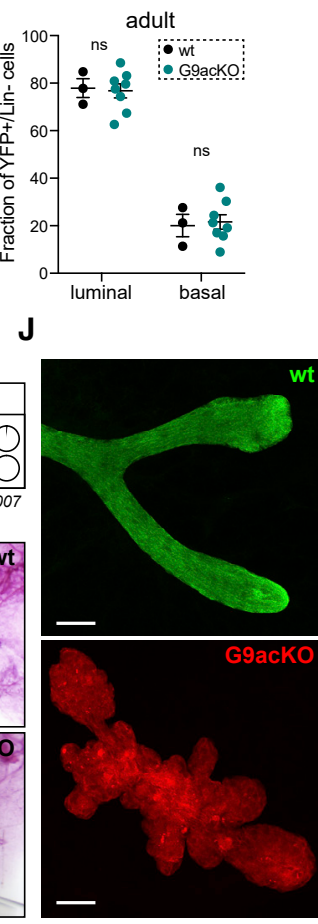

Figure 3. Mammary stem cell potential is impaired following G9a loss

(A-C) Representative FACS plots showing gating of luminal and basal cells $(A)$ and quantification of YFP-positive mammary epithelial cells (B) and luminal/basal cells (C) of pubertal (6-week-old) or adult (12-week-old) WT or G9acKO mice, as indicated. Data are mean \pm SEM. Pubertal mice, $n=5$ (WT), $n=3$ (G9acKO); adult mice, $n=3(\mathrm{WT}), \mathrm{n}=8$ (G9acKO). Two-sided Student's $\mathrm{t}$ test $(\mathrm{B})$ or two-way ANOVA with Dunnett multiple comparisons test (C).

(D-G) 2D (D and E) or 3D ( $F$ and G) colony formation assay of adult WT or G9acKO basal cells, showing representative images of three independent experiments (D and F) and quantification of colony area (E and G). Scale bars, $5 \mathrm{~mm}(\mathrm{D})$ and $50 \mu \mathrm{m}(\mathrm{F})$. In (E and G), data are mean $\pm \mathrm{SEM}$ of individual colonies pooled from triplicate wells. Two-sided Student's t test.

(H) Pie charts representing the percentage of mammary fat pad area filled by each cleared fat pad mammary transplant (5,000 mammary epithelial cells). Transplant recipients were WT mice. Transplant donor genotypes are indicated.

(I) Representative images of cleared fat pad transplants of the indicated genotypes (stitched images; STAR Methods). Scale bar, 2 mm.

(J) Immunofluorescence for endogenous FP (YFP or tdTomato, depending on the donor) to visualize cleared fat pad mammary transplant morphology. Scale bar, $50 \mu \mathrm{m}$. Color swap experiments were performed with equivalent results.

See also Figure S3.

transplant sorted total mammary epithelium from WT or G9acKO mice rather than basal cells only. All mice transplanted with 5,000 FACS-isolated WT mammary epithelial cells successfully developed mammary trees; in contrast, almost none of the mice transplanted with G9acKO cells did, and the few outgrowths that developed were small and displayed an abnormal morphology reminiscent of invasive organoids rather than ductal trees (Figures $3 \mathrm{H}-3 \mathrm{~J})$. These findings indicated that deletion of G9a resulted in a clear reduction of mammary stem cell potential that was at least in part cell autonomous.

\section{Lineage specification in G9acKO mammary epithelial cells is unaltered}

To test for lineage fidelity and specification, we analyzed individual WT or G9acKO mammary epithelial cells (sorted by FACS from virgin adults, YFP+/Lin-; Figure S3A) using singlecell RNA sequencing (scRNA-seq). We identified the previously described four cell clusters (Pal et al., 2017): basal, luminal progenitor (LP), luminal intermediate, and mature luminal (ML) cells (Figures 4A, S4A, and S4B). Notably, we also identified a fifth cluster adjacent to LP cells and defined by expression of cell cycle-related genes (Figure S4A; Table S1); we called these cells "proliferative LPs." The identities of the mammary cell populations were confirmed based on expression of lineage markers (Figure S4C). No major differences in the co-expression of the basal cell markers $\alpha \mathrm{SMA}, \mathrm{K} 14$, and p63 were observed, although the proportion of G9acKO basal cells expressing all three markers was reduced slightly (Figures S4D and S4E). Mammary epithelial cell clusters and lineage score expression (based on Pal et al., 2017) between WT and G9acKO mammary epithelial cells were largely unchanged (Figures $4 \mathrm{~B}$ and $\mathrm{S} 4 \mathrm{~F}$ ), except for a substantial reduction in proliferative LPs in G9acKO mammary epithelial cells (Figure 4A). Focusing on the basal cell cluster only, we identified a proliferative subcluster, again reduced in G9acKO mammary epithelia (Figure S4G; Table S2). Staining for the cell proliferation marker Ki67 confirmed a reduction in proliferation of luminal and basal G9acKO mammary lineages (Figures 4C, 2D, and S4H). We concluded that lineage fidelity and specification were essentially retained in G9acKO mammary epithelial cells. 
A

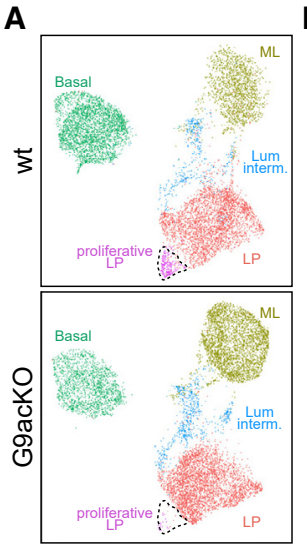

B

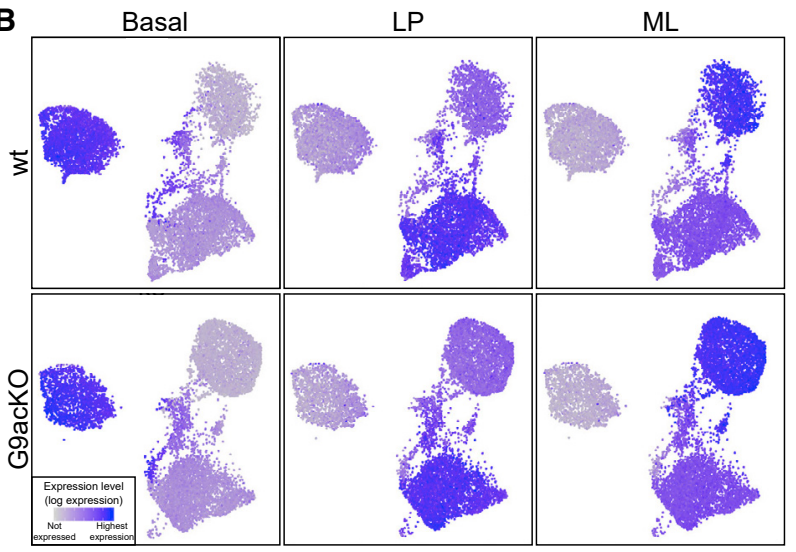

c

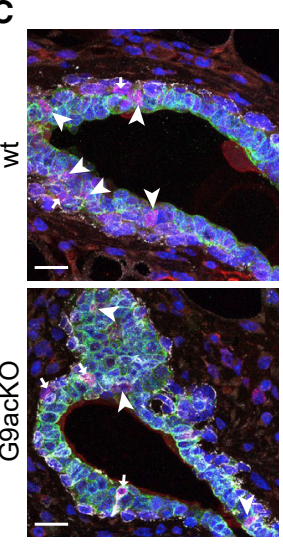

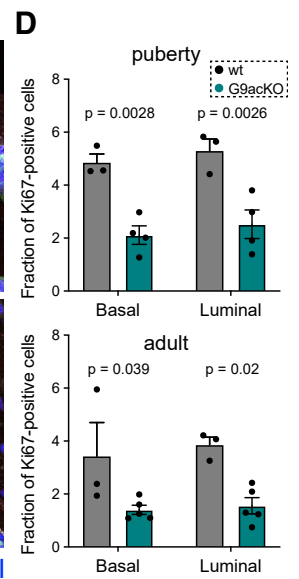

E
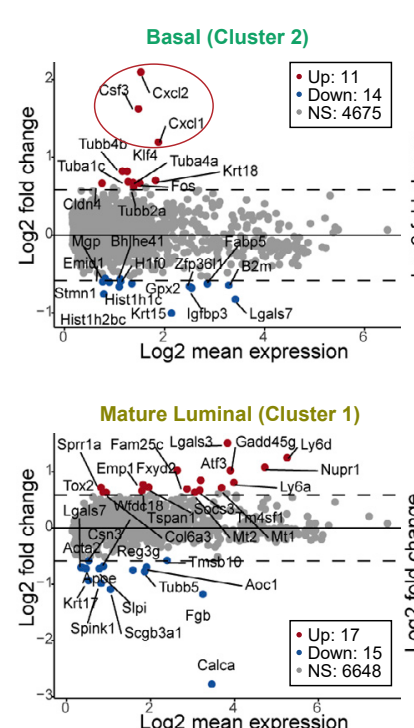

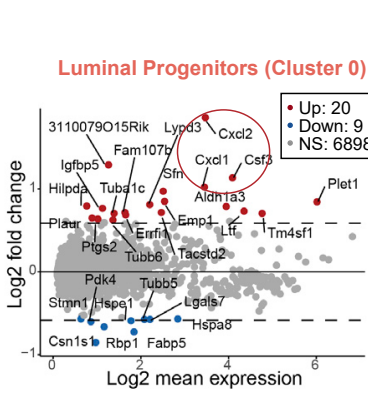

Luminal intermediate (Cluster 3)

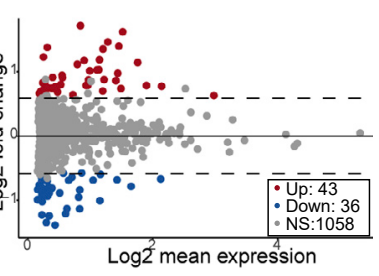

$\mathbf{F}$

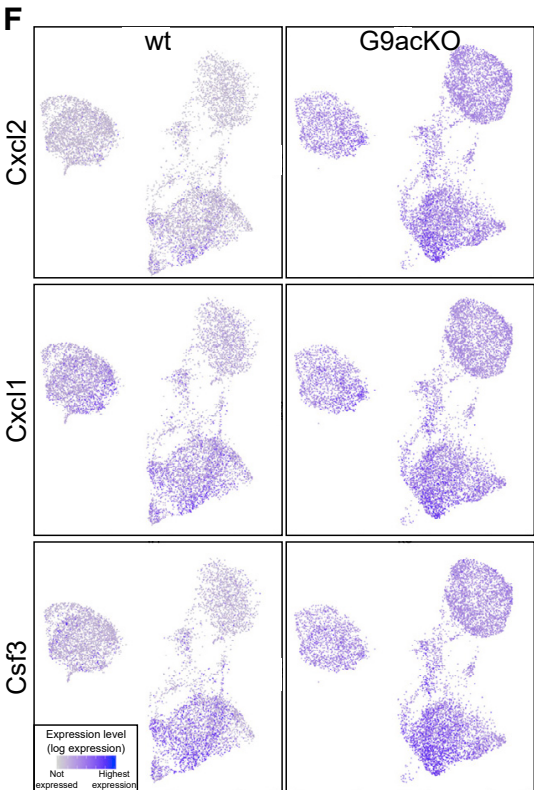

G

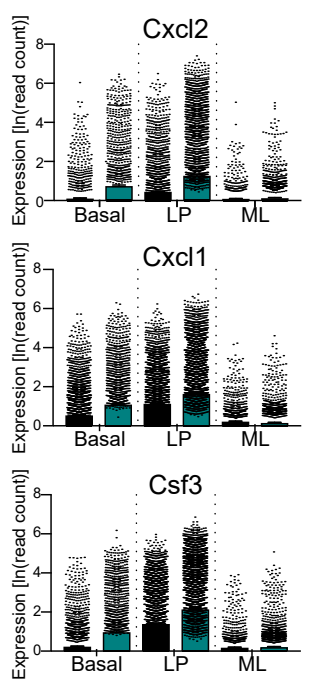

Figure 4. Correct lineage specification in G9acKO mammary epithelia

(A) UMAP plots of the transcriptomes of total epithelial cells of mammary glands from adult WT or G9acKO mice, generated using 10x Genomics, separated by genotype. $n=2$ mice per group. Major clusters (with the contribution of WT:G9acKO cells indicated) were basal (cluster 2, 3,226:1,760 cells), luminal progenitors (LPs; cluster 0, 3,338:3,648 cells), mature luminal (ML; cluster 1, 1,660:3,338 cells), luminal intermediate (cluster 3, 464:651 cells), and proliferative LPs (cluster 4, 251:23 cells).

(B) Expression of known lineage-specific signatures was tested in the epithelial cell clusters separated by genotype (STAR Methods) to confirm their identities. Color indicates the expression score of each indicated signature.

(C) Immunofluorescence for CK8 (green), Ki67 (red), and CK14 (gray) on mammary fat pads from adult WT or G9acKO mice. Nuclei were counterstained with DAPI (blue). $n=3$ (WT), $n=5$ (G9acKO) mice. Arrowheads indicate proliferative luminal cells; arrows indicate proliferative basal cells. Scale bar, $25 \mu \mathrm{m}$. For visualization, the brightness of the red channel was modified uniformly across all images. Quantification was performed on raw data.

(D) Quantification of Ki67-positive (proliferative) cells in (C). Shown are cells in which Ki67 colocalizes with cytokeratin 14 (proliferative basal cells) or CK8 (proliferative luminal cells). Data are mean \pm SEM of individual animals. For pubertal mice, $n=3$ (WT), $n=4$ (G9acKO); for adult mice, $n=3$ (WT), $n=5$ (G9acKO). Two-way ANOVA with Dunnett multiple comparisons test.

(E) MA plots highlighting DEGs between WT and G9acKO cells by cluster. Genes with FDR $<0.05$ and FC $>1.5$ are indicated for basal, LP, and ML cells (Table S2). Highly differentially expressed cytokines are highlighted.

(F) Expression of differentially expressed cytokines to determine their patterning among the cell clusters. Color indicates the expression level.

(G) Quantification of (E). For statistics, see the DEG analysis in (E) and Table S2.

See also Figure $S 4$.

Strikingly, few genes $(25,29$, or 32$)$ were differentially expressed for the basal, LP, or ML cell clusters, respectively, comparing G9acKO with the WT (false discovery rate [FDR] < 0.05 , fold change $[F C]>1.5$; Table S3). The genes upregulated in G9acKO versus WT basal cells included the transcriptional repressor of mesenchymal genes Klf4 (Tiwari et al., 2013), keratin 18 (which is typically luminal), and several cytoskeleton-associated genes (Tuba1c, Tuba4a, Tubb2a, and Tubb4b) (Figure 4E), 
A

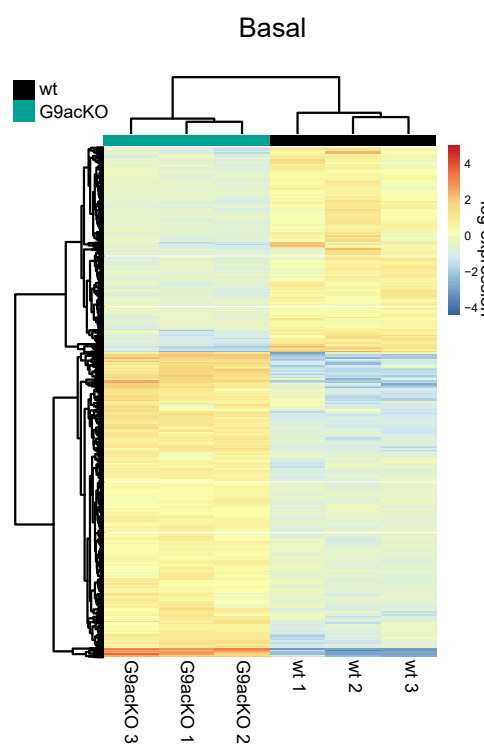

C

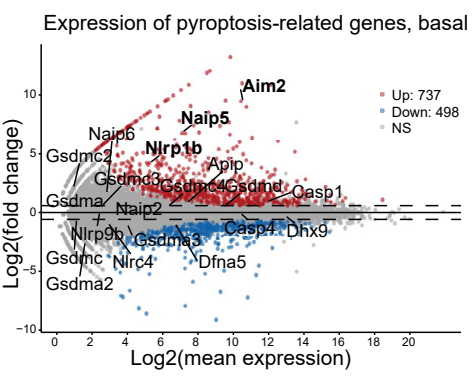

B

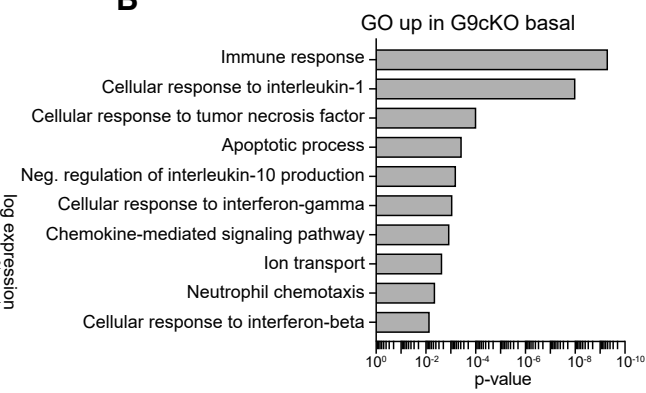

GO down in G9cKO basal

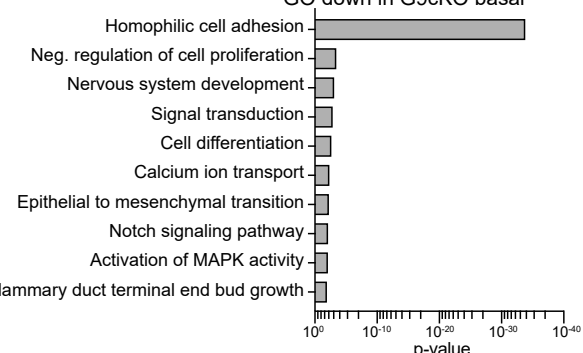

D
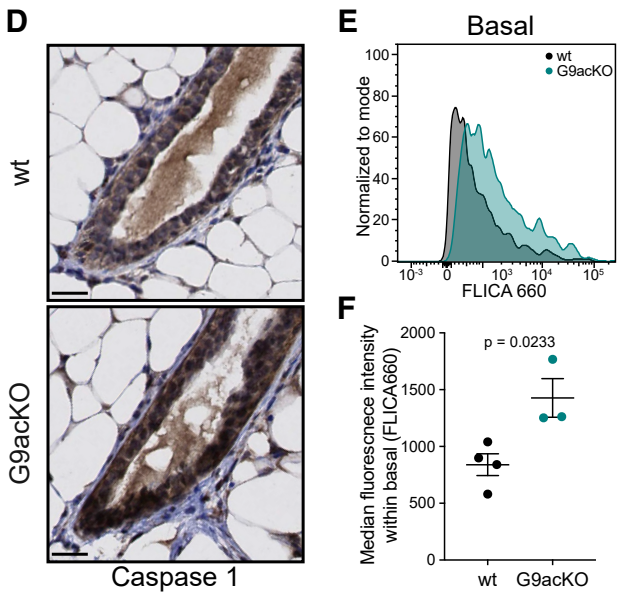

F

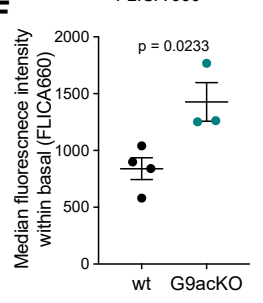

I

H Inflammatory monocytes
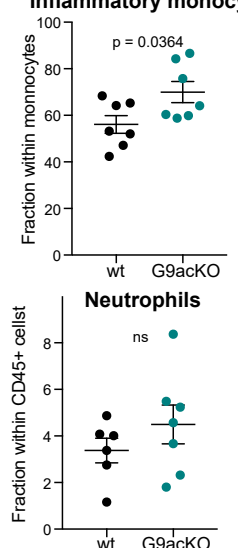

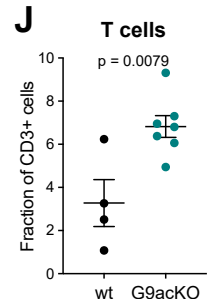

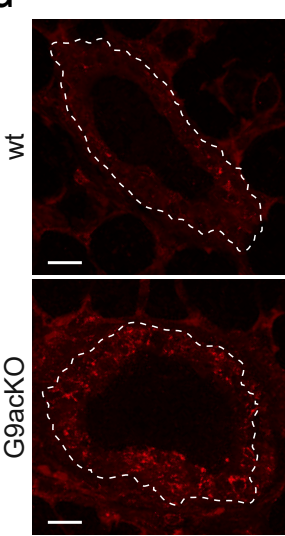

dsDNA
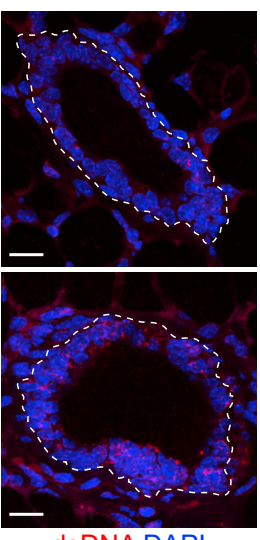

dsDNA DAPI 


\section{$\infty$ CellPress}

\section{Cell Stem Cell}

consistent with the columnar (epithelial) appearance of G9acKO basal cells. Our single-cell-level expression data suggested that upregulation of these genes was the result of increased median expression levels per cell and a slight expansion of a basal population already present in basal WT cells (data not shown). Notably, the cytokine genes Cxc/1, Cxcl2, and Csf3 were upregulated strongly in basal and LP G9acKO cells (Figures 4E$4 G)$, suggesting a potential paracrine effect of G9a deletion.

\section{A skewed immune environment in the G9acKO mammary gland}

To gain higher resolution of differentially expressed genes (DEGs) after G9a deletion, we performed bulk RNA-seq of FACS-isolated mammary luminal and basal epithelial cells from virgin adult WT or G9acKO mice. We found a total of 1,235 DEGs (498 down- and 737 upregulated) in basal cells and 683 DEGs (92 down- and 590 upregulated) in luminal cells (FDR < $0.05, \mathrm{FC}>1.5$; Figures 5A and S5A; Table S4). Gene Ontology (GO) analysis of upregulated DEGs in G9acKO cells indicated strong activation of immune response pathways (Figures 5B and S5B; Table S4), with numerous cytokines and chemokines misexpressed in luminal and basal cells (Table S4), consistent with the scRNA-seq results (Figures 4E-4G). For instance, the GO categories "cellular response to interleukin- 1 " and "cellular response to interferon $\gamma / \beta$ " were overrepresented in basal cells, whereas "cytokine-cytokine receptor interaction" and "graft versus host disease" were the most significantly overrepresented KEGG pathways (Table S4). Luminal cells showed upregulated DEGs in the GO category "pyroptosis," an immunogenic type of programmed cell death (Jorgensen et al., 2017; Shi et al., 2017), and key pyroptotic genes (e.g., Aim2, Casp1, Gsdmc2, Naip5, Naip6, and NIrp1b) were upregulated in luminal and/or basal G9acKO cells (Figures 5C and S5C). Finally, array-based whole-genome expression profiling of pubertal WT or G9acKO luminal and basal cells (Figure S5D; Table S5) showed a substantial overlap in DEGs between puberty and adulthood, notably including the pyroptosis-related Aim2, Casp1, and Naip5 genes (Figure S5E). We concluded that these DEGs characterized the mammary epithelium of G9acKO mice in general rather than a specific developmental time point. The gene for G9a, Ehmt2, was not detected as a DEG by the Affymetrix gene expression arrays because of probe targeting to Ehmt2 regions retained in G9acKO animals. Loss of Ehmt2 expression was confirmed by real-time quantitative PCR specific for the deleted exon 27 of Ehmt2 (Figure S5F).

Caspase-1 (the pyroptosis effector caspase) had elevated RNA, protein, and activity levels in G9acKO mammary epithelium versus WT (Figures 5D-5F and S5G-S5I), indicating activation of a functional inflammasome upon G9a loss. Caspase-1 is activated by diverse triggers, including viruses, bacteria, and toxins (Jorgensen et al., 2017); however, co-upregulation of the pyroptotic sensor protein Aim2, which specifically senses cytosolic double-stranded DNA (dsDNA) (Fernandes-Alnemri et al., 2009; Hornung et al., 2009), suggested cytosolic dsDNA as a trigger. Interestingly, the GO categories "positive regulation of MDA-5 signaling pathway" and "positive regulation of RIG-I signaling pathway" were significantly underrepresented in G9acKO versus WT luminal cells (Figure S5B; Table S4). The MDA-5 and RIG-I signaling pathways form part of the cytosolic DNA sensing machinery of the cGAS-STING pathway (Zevini et al., 2017), which has been reported to be negatively regulated by Aim2 inflammasome-based pyroptotic cytosolic DNA sensing (Corrales et al., 2016; Nakaya et al., 2017).

Histological staining revealed that cytosolic dsDNA levels were strongly elevated in luminal and basal cells in G9acKO versus WT mammary epithelium (Figures $5 \mathrm{G}$ and S5J). We ruled out that this cytosolic dsDNA came from bacterial or viral infection (mice were kept in specific pathogen-free conditions, and same-cage littermates were used as controls), suggesting self-DNA as a potential source. Notably, cell death levels were unchanged (Figures S5K and S5L), suggesting that G9a loss only triggered altered cytokine expression but not pyroptotic cell death. Similarly, Aim2-inflammasome activation in the epidermis leads to cytokine production but not pyroptotic cell death (Naik et al., 2017).

Finally, consistent with pyroptosis-like inflammatory signaling, we observed a slight but significant increase in infiltrating inflammatory monocytes, a (non-significant) increase in infiltrating neutrophils, and an increase in infiltrating T lymphocytes in G9acKO mice versus WT littermates (Figures $5 \mathrm{H}-5 \mathrm{~J}$ ). There were no differences in infiltration of B cells, macrophages, or natural killer (NK) cells (data not shown).

\section{Lack of G9a results in chromatin opening in LTR retrotransposon regions}

To assess whether reduced H3K9me2 levels caused by G9a loss led to increased chromatin opening, we performed an assay for transposase-accessible chromatin with high-throughput sequencing (ATAC-seq) (Buenrostro et al., 2013) on FACS-isolated luminal or basal cells from WT or G9acKO mice (Figure S3A). About $65 \%(8,445$ of 12,857$)$ or $81 \%(2,138$ of 2,638$)$ of differentially open chromatin regions were more accessible in G9acKO basal or luminal cells, respectively, as compared to WT controls (Figures 6A and S6A). G9acKO differentially open chromatin regions were, on average, longer than those in WT cells (Figures $6 \mathrm{~B}$ and $\mathrm{S} 6 \mathrm{~B}$ ) and were enriched in intergenic

(D) Immunohistochemistry for caspase-1 on mammary fat pads from adult WT or G9acKO mice. Nuclei were counterstained with hematoxylin. Scale bar, $25 \mu$ m. $\mathrm{n}=5(\mathrm{WT}), \mathrm{n}=4$ (G9acKO) mice. Representative images are shown.

(E) Representative FACS plot of the fluorochrome-labeled inhibitors of caspases (FLICA) caspase-1 activity assay in WT or G9acKO basal cells. $n=4$ (WT) and $n=$ 3 (G9acKO) mice.

(F) Quantification of (E). Data are mean \pm SEM for individual animals. Two-sided Student's t test.

(G-J) Analyses of WT or G9acKO mammary fat pads from adult mice, showing (G) immunofluorescence for dsDNA (red) with nuclei counterstained with DAPI (blue). Representative images are shown. $n=5(\mathrm{WT}), \mathrm{n}=4$ (G9acKO) mice. Shown in $(\mathrm{H})$ is quantification of infiltrating inflammatory monocytes ( $\mathrm{n}=7$ ) or neutrophils ( $n=6$ [WT], $n=7$ [G9acKO]). Shown in (I) is immunohistochemistry for CD3, with nuclei counterstained with hematoxylin. Arrowheads indicate CD3positive lymphocytes within the mammary epithelium. Representative images are shown. $n=4(W T), n=7(G 9 a c K O)$ mice. Shown in (J) is quantification of (I). For (G) and (I): scale bar, $25 \mu \mathrm{m}$. For $(\mathrm{H})$ and $(\mathrm{J})$ : two-sided Student's t test; data are mean \pm SEM of individual animals.

See also Figure S5. 
A

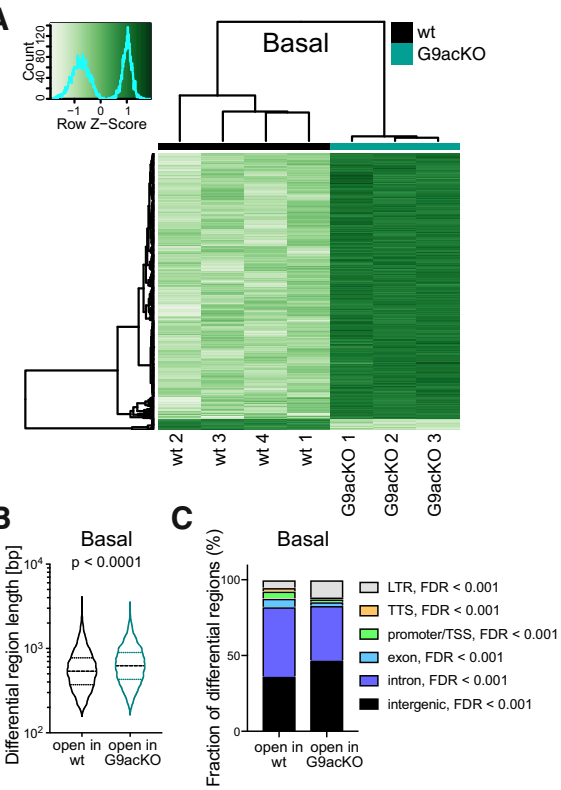

D

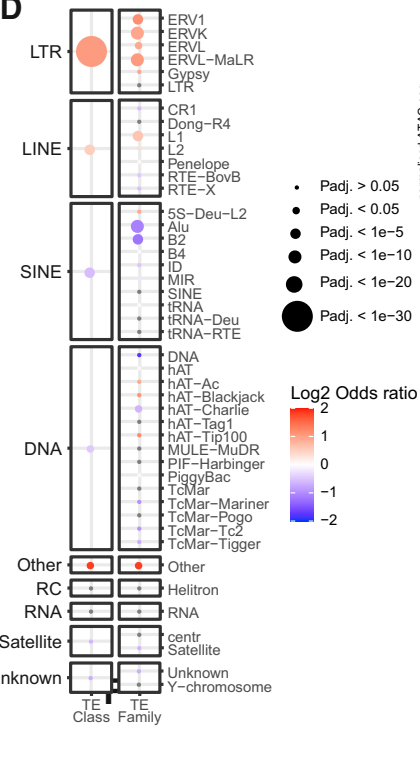

E

RLTR10D2

Basal ATAC G9acKO Basal ATAC wt Mappability score
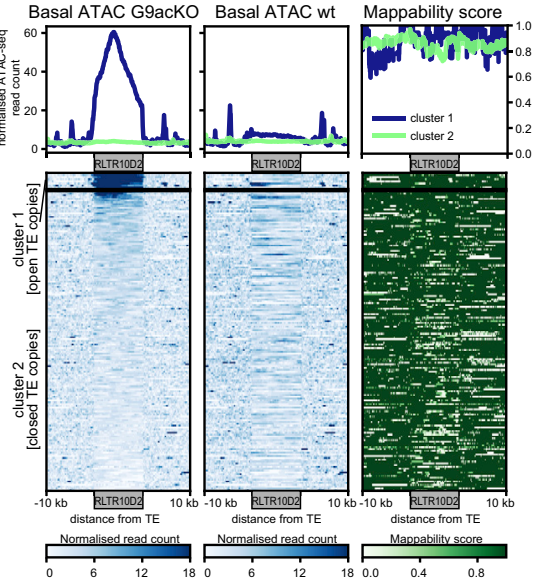

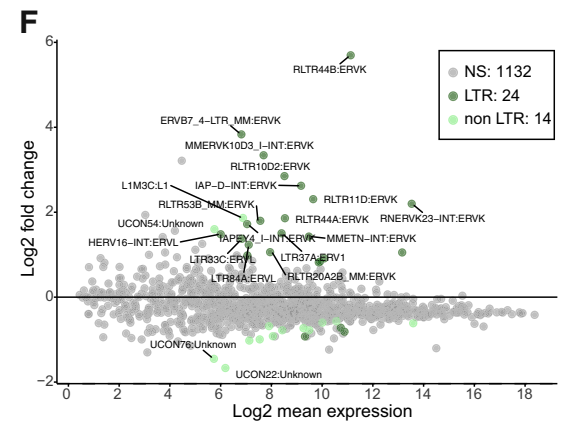

H

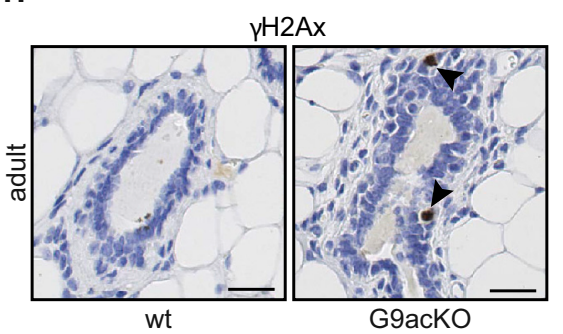

G chr10:38,058,186-38,091,428

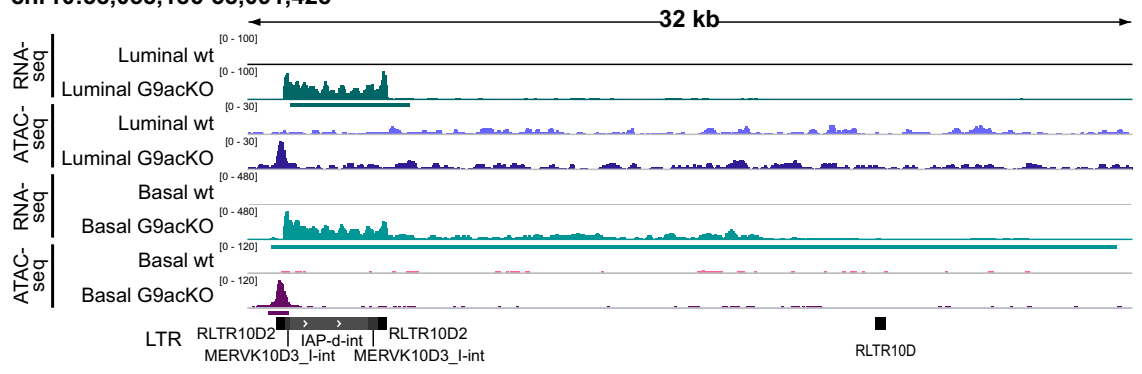

I

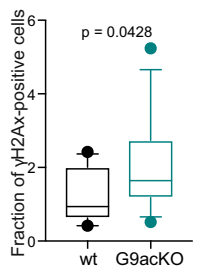

J

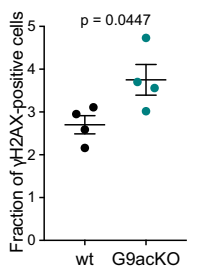

$\mathbf{K}$

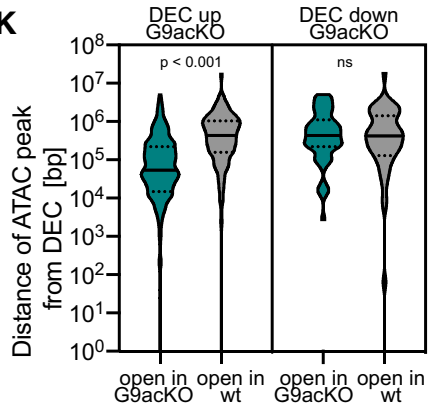

Figure 6. Increased chromatin opening in G9acKO mammary epithelium correlates with derepression of LTRs (A-G) Analysis of FACS-isolated WT or G9acKO basal cells, showing (A) correlation heatmap of regions of differential chromatin opening, (B) quantification of the length of differentially open chromatin regions, (C) annotation of the location of differentially open chromatin regions, (D) dot plot of enrichment of TEs within differential ATAC peaks, and (E) heatmap showing ATAC-seq read coverage of individual copies of the RLTR10D2 subfamily of LTR retrotransposons. Each row represents an individual RLTR10D2 locus $( \pm 10 \mathrm{~kb})$. RLTR10D2 copies that are in open chromatin (cluster 1) or closed chromatin (cluster 2) are indicated. The mappability scores of the individual loci demonstrate that differences in ATAC-seq read coverage are not due to differences in regional mappability. In (F), an MA plot shows differentially expressed TEs; significant ones (ajd. $p<0.01$ ) are highlighted in green and subclassified as LTR or non-LTR. Shown in (G) is an example of increased chromatin accessibility leading to full-length IAP derepression that results in pervasive transcription.

(H) Immunohistochemistry for $\gamma \mathrm{H} 2 \mathrm{AX}$ on mammary fat pads from adult (WT or G9acKO) mice. Nuclei were counterstained with hematoxylin. Scale bar, $25 \mu \mathrm{m}$. $\mathrm{n}=$ 4 mice per group. Representative images are shown.

(I) Quantification of (E). Each data point indicates an ROI; 3 ROls per mouse were used.

(J) Quantification of $\gamma \mathrm{H} 2 \mathrm{AX}$ staining of WT or G9acKO mammary epithelial cells by flow cytometry. Each data point represents an individual animal. $n=4$ per group.

(K) Violin plot of the distance between differentially expressed clusters (DECs) and the closest differentially open chromatin region in basal cells, highlighting an association between G9acKO DEC upregulation and chromatin opening.

In (B), (I), and (J), two-sided Student's t test; in (C), Fisher's exact test with FDR correction for multiple testing. See also Figure S6. 


\section{$\infty$ CellPress}

regions and regions containing the LTR retrotransposon class of transposable elements (TEs) (Figures 6C and S6C). In contrast, in WT luminal and basal cells, differentially open regions were predominantly in genic (promoter/exon/intron/transcription termination site [TTS]) regions (Figures $6 \mathrm{C}$ and $\mathrm{S} 6 \mathrm{C}$ ). This suggests that deletion of G9a preferentially opened intergenic chromatin regions containing LTR retrotransposons, which, when derepressed, could be reverse transcribed through a dsDNA intermediate in the cytosol and trigger the Aim2 inflammasome signaling cascade (Johnson, 2019).

To test this hypothesis, we analyzed the enrichment of different classes of TEs in differential ATAC peaks from WT or G9acKO mammary epithelial cells. Strikingly, compared with WT cells, differentially open chromatin regions in G9acKO basal cells were enriched for LINE and LTR retrotransposon classes of TEs, most prominently of the ERVK and ERVL-MaLR TE families (Figures 6D). LINE and LTR retrotransposon classes of TEs were also overrepresented in chromatin regions open differentially in G9acKO luminal cells, but no single TE family was enriched significantly (Figure S6D). We saw no examples where G9a loss resulted in increased chromatin accessibility at the loci of all copies of any individual TE; for instance, less than $10 \%$ of copies of the RLTR10D2 subfamily of TEs overlap with differentially accessible chromatin (Figures 6E and S6F). This suggests that $\mathrm{H} 3 \mathrm{~K} 9 \mathrm{me} 2$ on its own is not a general mechanism of repression of any TE in mammary epithelial cells but works in concert with other transcriptional and epigenetic mechanisms to establish stable repression of TEs.

\section{LTR retrotransposons are transcriptionally derepressed in G9acKO mice}

Re-visiting the total RNA-seq data (Figure 5), we observed differential expression of 20 and 38 TEs (predominantly LTR retrotransposons) in luminal and basal G9acKO cells, respectively (FDR < 0.01) (Figures 6F and S6G). Four LTR retrotransposons from the ERVK family (RLTR44A, RLTR53B, RLTR10D2, and RLTR44B) were upregulated and highly enriched for differential chromatin opening in basal and/or luminal G9acKO mammary epithelial cells (Figure S6H). Thus, chromatin opening in TE regions led to their transcriptional derepression in G9acKO mammary epithelial cells.

To determine whether the derepressed LTR retrotransposons in G9acKO cells were full length (i.e., functional and able to generate cytosolic dsDNA), we mapped their genomic locations and compared these with annotations of full-length LTR retrotransposon subfamilies (as available). We used DERfinder (STAR Methods) to identify differentially expressed clusters (DECs) along the genome. Focusing on intergenic DECs (to reduce noise from gene expression), we found a total of 777 intergenic DECs in basal G9acKO cells (725 up- and 52 downregulated; median length, 1,867 bp; 95\% confidence interval [Cl], 1,490-2,158 bp) and 174 intergenic DECs in luminal G9acKO cells (160 up- and 14 downregulated; median length, 2,121 bp; 95\% Cl, 1,548-2,904 bp) (FDR < 0.05; Table S6). By mining the TEs annotated to upregulated DECs for internal LTR retrotransposon regions flanked by LTRs to identify fulllength LTR retroviral elements, we found that the MMVL30INT and IAP-D-INT internal regions flanked by LTRs were derepressed repeatedly in G9acKO basal and luminal cells versus the WT (Figures 6G and S6I). MMVL30-INT is the internal region of the VL30 retrovirus subfamily, which accumulates as cytosolic dsDNA when derepressed and triggers an antiviral immune response (Herquel et al., 2013). Notably, 18 of 86 known full-length VL30 genomic copies (Markopoulos et al., 2016) were derepressed in G9acKO mammary epithelium versus the WT, highlighting the VL30 subfamily of retroviruses as a potential source of cytosolic dsDNA in G9acKO mammary epithelia. Full-length retroviral activation can also lead to genome re-integration, which triggers DNA damage in the host cell (Johnson, 2019). Indeed, we observed that the DNA damage marker $\gamma \mathrm{H} 2 \mathrm{Ax}$ was increased in G9acKO mammary epithelia versus the WT (Figures 6H-6J, S6J, and S6K).

Notably, upregulated DECs were closer to differentially opened chromatin regions in G9acKO than in WT epithelial cells (Figures $6 \mathrm{~K}$ and $\mathrm{S} 6 \mathrm{~L}$ ). These results suggest that DEC (and thus TE) derepression in G9acKO cells is regulated by de novo chromatin opening, which could cause the altered cytokine and inflammatory milieu in G9acKO fat pads.

\section{Aim2 upregulation depends on TE derepression and is} responsible for developmental delay of G9a-null mammary epithelium

LINE, RLTR44A, and MERVK10D3 TE upregulation was maintained in vitro in G9acKO mammary epithelial cells compared with WT controls but abrogated following treatment with lamivudine (a nucleoside reverse-transcriptase inhibitor; Figure S7A), concomitant with reduced cytosolic dsDNA levels (Figure S7B). Lamivudine treatment also abolished upregulation of Aim2 and Casp1 (Figure S7C) in G9acKO compared with WT mammary epithelial cells. Thus, TE derepression and cytosolic dsDNA accumulation are functionally linked to activation of the Aim2-dependent inflammasome in G9acKO mammary epithelial cells. We therefore next wanted to find out whether disrupting the pyroptotic pathway in vivo could ameliorate the G9acKO mammary developmental defects. We injected shCTR or shAim2 lentiviral particles intraductally (through the nipple) in early-pubertal (4-week-old) WT or G9acKO mice and assessed mammary outgrowth after 2 weeks in pubertal mice. shAim2 knockdown (Figure S7D) rescued mammary fat pad invasion defects but not the distended TEB phenotype of G9acKO mice and had no effect on WT littermates (Figures 7A and 7B). These data indicated that the dsDNA sensor Aim2 and its related activation of the pyroptotic pathway were responsible, at least in part, for the G9acKO ductal invasion defects.

\section{Adaptive immune response underlying G9acKO} mammary stem cell defects

To test whether an autoimmune-like environment in G9acKO mammary fat pads affected the cleared mammary fat pad repopulation potential of G9acKO mammary epithelial cells, we transplanted 5,000 FACS-isolated, YFP-positive WT or G9acKO mammary epithelial cells into immunocompromised athymic nude mice. In contrast to transplants into immunocompetent WT recipients (Figures $3 \mathrm{H}-3 \mathrm{~J}$ ), more than half of the immunocompromised fat pads supported outgrowth of organized G9acKO mammary tree structures, with two fat pads even being fully populated (Figures 7C and 7D). Nonetheless, the G9acKO 
A

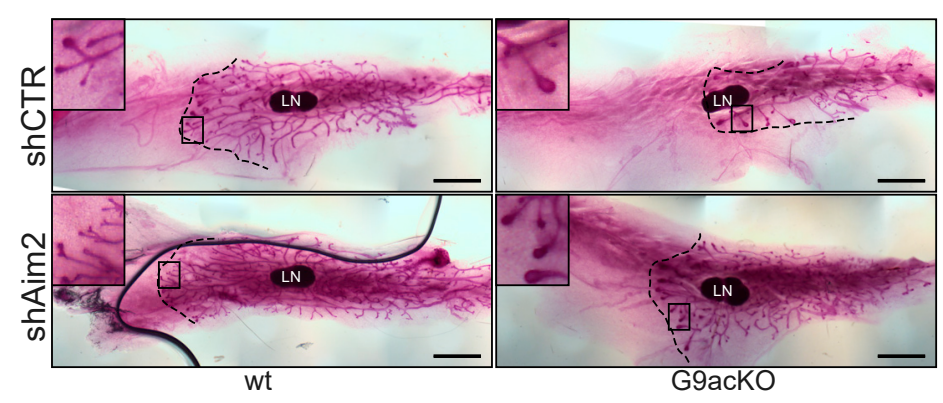

B

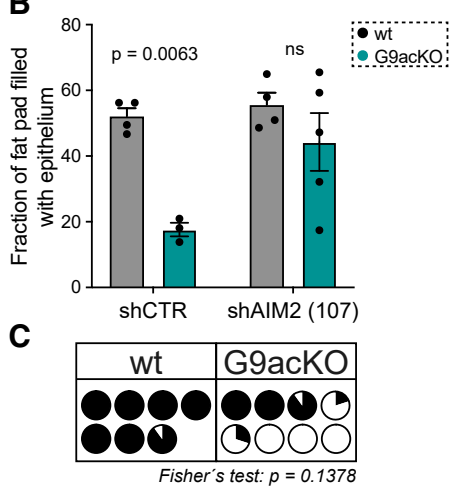

D

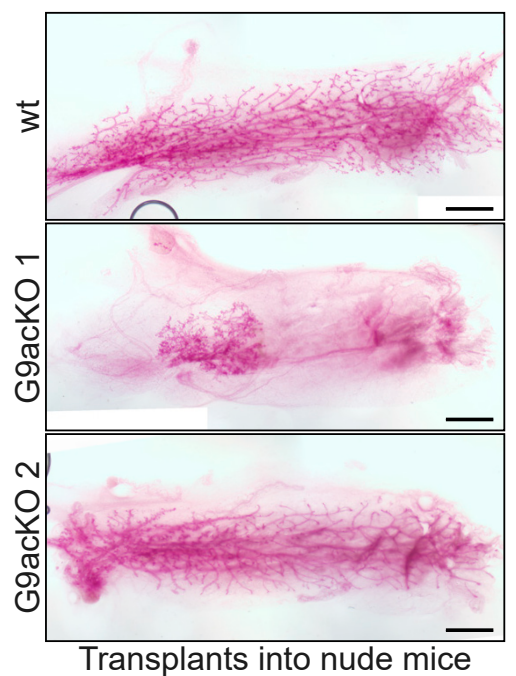

E

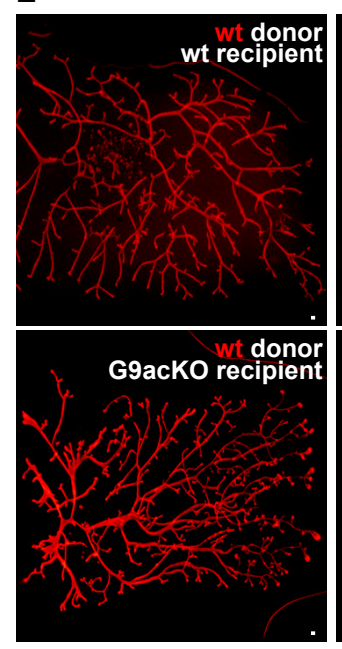

F

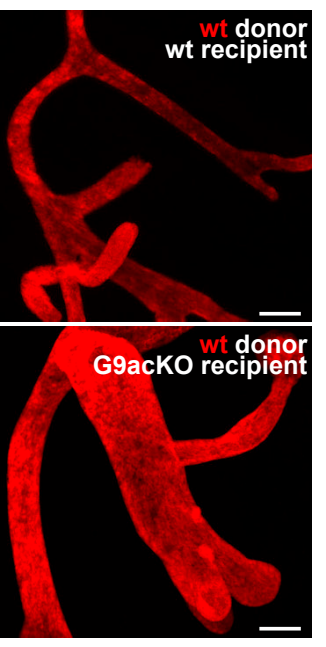

G
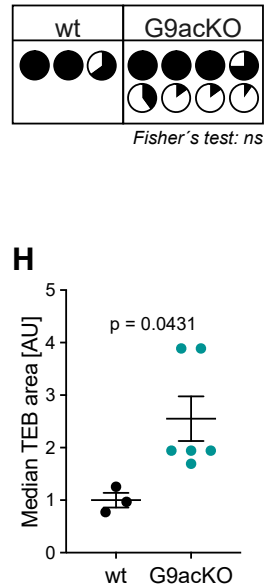

Figure 7. Paracrine deregulation contributes to G9acKO morphological defects

(A) Carmine-alum staining of mammary wholemounts from pubertal WT or G9acKO mice injected intraductally with shCTR or shAim2 lentiviral particles (stitched images; STAR Methods). The dashed line indicates the mammary tree invading front. LN, lymph node. Scale bar, $2 \mathrm{~mm}$.

(B) Quantification of (A). Data are mean \pm SEM of individual fourth mammary fat pads. $n=4$ (WT), $n=3$ (G9aKO shCTR), and $n=5$ (G9acKO shAim2). Two-way ANOVA with Dunnett multiple comparisons test.

(C and D) Representative images (D; stitched images; STAR Methods) and quantification of percentages of filled areas (C) of cleared mammary fat pad transplants of WT or G9acKO mammary epithelial cells into nude mice. Transplant donor genotypes are indicated. Fisher's exact test. Scale bar, 2 mm.

(E and F) Representative immunofluorescence images of endogenous tdTomato, to visualize fat pad transplant morphology from WT donors. Transplant recipient genotype is indicated. Scale bar, $100 \mu \mathrm{m}$.

(G) Pie charts representing the percentage of area filled by donor mammary epithelium (tdTomato-positive) in each individual non-cleared fat pad mammary transplant. Fisher's exact test.

$(\mathrm{H})$ Quantification of the median terminal end bud (TEB) area of (E). Data are mean \pm SEM of individual transplants. $n=3$ for WT recipients, $n=6$ for $G 9 a c K O$ recipients. Outgrowths (rudimentary organoid-like mammary structures with no TEBs) in two G9acKO recipients were not considered. Two-sided Student's t test. See also Figure S7.

luminal and basal stem cell potential was impaired severely in vitro (Figures 3D-3G and S3C-S3H). Therefore, we concluded that reduced G9acKO stem cell potential is the result of cellautonomous and non-autonomous effects in G9acKO mammary fat pads.

\section{Normal mammary morphogenesis is disrupted by} changes in the stromal mammary milieu in the absence of G9a

To determine the influence of the altered cytokine milieu and collagen content in G9acKO fat pads on morphology, we transplanted WT mammary epithelial cells into non-cleared fat pads of
WT or G9acKO recipients (non-cleared recipient fat pads allowed continuous niche conditioning by the endogenous mammary epithelium). Indeed, although WT cells transplanted into WT recipients appeared normal, WT cells transplanted into G9acKO fat pads displayed distended TEBs, thickened ducts, and increased lateral branching, partially recapitulating the G9acKO phenotype (Figures 7E-7H). Therefore, we conclude that the defects observed in development of the mammary epithelium with G9a loss consisted of cell-autonomous components (i.e., decreased stem cell potential; Figure 3) as well as cell non-autonomous components (i.e., altered paracrine signaling). 


\section{$\infty$ CellPress}

\section{Cell Stem Cell}

\section{DISCUSSION}

Whether chromatin accessibility in non-coding regions plays a role in determining cell fate and tissue development is not known. We show that the histone methyltransferase G9a is indispensable for mammary gland function and organization of the stem cell pool but not required for mammary epithelial cell fate determination. We provide evidence of cell-autonomous (i.e., decreased stem cell potential) and cell non-autonomous (i.e., paracrine signaling, extracellular matrix deposition, and immune cell recruitment) aspects of the observed defects. We suggest that these phenotypes are due to derepression of specific TEs that, when activated, trigger an antiviral immune response. This altered immune environment even precludes outgrowth of WT mammary cells transplanted into the mammary fat pads of G9acKO mice.

We previously reported a substantial decrease in stem cell potential following G9a loss in epidermal stem cells (Avgustinova et al., 2018). Intriguingly, epidermal stem cells devoid of G9a also upregulated the expression of genes involved in an antiviral innate immune response, such as Aim2. However, in stark contrast to the severely aberrant G9acKO mammary glands reported here, epidermal development, morphology, and maintenance were largely unscathed, and gene expression was not majorly altered, in mice lacking G9a in epidermal cells. This striking difference in the effect of G9a loss between the epidermis and mammary gland might be explained by the ability of the epidermis (as a consequence of its natural barrier function) to respond to pathogens (e.g., viruses) without altering its development or homeostasis. For instance, hair follicle stem cells exposed to inflammatory stress retain an epigenetic memory of pyroptotic genes, such as Aim2 and Casp1, that allows them to respond more efficiently to exposure to subsequent rounds of inflammatory stress (Naik et al., 2017).

G9acKO mammary epithelia are disorganized and multilayered (Figures 1J-1N and S1D-S1G), reminiscent of hyperproliferative lesions. Formation of such hyperplastic lesions is often associated with an imbalance between cell proliferation and cell death in favor of increased proliferation. However, G9acKO luminal and basal cells are less proliferative than their WT counterparts (Figures 4C, 4D, S4G, and S4H), arguing against neoplastic transformation causing the G9acKO mammary duct polarity defects. Consistently, G9acKO mice do not develop spontaneous mammary tumors even during aging (up to 95 weeks; data not shown). Although we cannot exclude that the altered inflammatory environment in G9acKO mice precludes development of bona fide malignant lesions from a pre-neoplastic state, we propose that the aberrant G9acKO mammary tissue polarity and multilayering stem from increased tissue stiffness, which hinders TEB outgrowth and is evidenced by increased collagen deposition in G9acKO compared with WT mammary fat pads (Figures 2B-2D and S2C-S2E). Consistently, WT mammary ducts that develop following transplantation into G9acKO hosts display larger TEBs than those transplanted into WT recipients (Figures 7F and 7H). We conclude that the G9acKO stromal milieu is in part responsible for the morphological defects observed in G9acKO mammary glands.

Surprisingly, in light of the severe morphological defects, the cell fate specification of the G9acKO mammary epithelium re- mained largely intact; the principal changes were lower proportions of proliferative basal and LP populations (Figures $4 \mathrm{~A}$ and S4; Tables S1 and S2). The slight reduction of ER $\alpha$-positive luminal cells in G9acKO mammary ducts (Figures S2F and $\mathrm{S} 2 \mathrm{G}$ ) could be partially responsible (through a decrease in proproliferative signals) for the reduced proliferative LP cells; however, this is unlikely the only mechanism because $E R \alpha$-negative G9acKO basal and epidermal cells also displayed reduced proliferative capacity (Figures 4D and S4G; Avgustinova et al., 2018).

Although DNA methylation is considered the principal mechanism of TE repression in higher eukaryotes, DNMT-independent mechanisms have also been reported (Hutnick et al., 2010), including H3K9 methylation and heterochromatin formation (Karimi et al., 2011; Liu et al., 2014; Matsui et al., 2010). H3K9me2 has been implicated in L1 germline repression (Di Giacomo et al., 2014) as well as in MERVL repression in embryonic stem cells (Maksakova et al., 2013) and in ovarian cancer epithelial cells (Liu et al., 2018). Mechanistically, TEs (and LTR retrotransposons in particular) can act as cis-regulatory "promotor" elements to drive gene expression (Burns, 2017; Chuong et al., 2017); however, this effect was negligible for LTR retrotransposon derepression in G9acKO mammary epithelial cells (Table S6). Full-length TEs can be detected by the cell as non-self DNA, triggering an inflammatory response (Chuong et al., 2017; De Cecco et al., 2019). Indeed, in G9acKO mammary epithelial cells, we showed altered cytokine and chemokine gene expression, extracellular matrix (ECM) deposition, and immune cell infiltration in addition to LTR retrotransposon derepression. Immune cells are active participants in mammary gland development through secretion of matrix metalloproteases and tissue inhibitors of matrix metalloproteases (Reed and Schwertfeger, 2010; Tan et al., 2014), which affect TEB morphology and branching morphogenesis (PageMcCaw et al., 2007; Wiseman et al., 2003). This is consistent with the mammary defects in G9acKO mice and the aberrant mammary structures formed by WT mammary epithelial cells in G9acKO mice.

Our results suggest that aberrant activation of TEs prevents proper development of the mammary gland. This phenotype might be related to derepressed TEs associated with age-related inflammation (Benayoun et al., 2019; De Cecco et al., 2019), which may contribute to cancer initiation (Burns, 2017; Saini et al., 2020). Future work is necessary to determine the precise mechanisms that regulate G9a-mediated TE repression during development and whether and how these are deregulated during aging and cancer.

\section{Limitations of study}

G9a represses ERVL elements in embryonic stem cells (ESCs) (Maksakova et al., 2013), ERVL and ERVL-MaLR elements in mouse epiblasts (Zylicz et al., 2015), and L1 elements in spermatogonia (Di Giacomo et al., 2014). Although we observed that the ERVL, ERVL-MaLR, and L1 TE families were significantly enriched in differentially open chromatin regions in G9acKO basal cells (Figure 6D), we observed no differential RNA expression of these TE families (Figures $6 F$ and S6G). Thus, we suggest that G9a contributes to ERVL/ERVL-MaLR/L1 TE repression, but that continued transcriptional repression in homeostatic mammary epithelial cells is maintained by additional repressive 
mechanisms (e.g., DNA and/or histone methylation) upon G9a loss. Elucidating the underlying molecular intricacies of these TE-repressive mechanisms in addition to G9a activity requires future studies.

\section{STAR $\star$ METHODS}

Detailed methods are provided in the online version of this paper and include the following:

- KEY RESOURCES TABLE

- RESOURCE AVAILABILITY

O Lead contact

○ Materials availability

O Data and code availability

- EXPERIMENTAL MODEL AND SUBJECT DETAILS

Mouse strains

$\bigcirc$ Cell lines

- METHOD DETAILS

O Clonogenic assays

S Single-cell preparation

O Flow cytometry

O Mammary transplants

O Lentiviral production

O Intraductal lentiviral injections

O Caspase activity assay

○ Mammary wholemounts

O Immunostaining

O ATAC sequencing

O Whole-genome expression analysis

O Real-time quantitative PCR (RT-qPCR)

O Single-cell RNA-sequencing (scRNA-seq)

O scRNA-seq analysis

O Transposable element differential expression analysis

- Differential ATAC-seq peak enrichment analysis

- QUANTIFICATION AND STATISTICAL ANALYSIS

\section{SUPPLEMENTAL INFORMATION}

Supplemental information can be found online at https://doi.org/10.1016/j. stem.2021.04.030.

\section{ACKNOWLEDGMENTS}

S.A.B. was supported by the European Research Council (ERC) and the Government of Cataluña (SGR grant). C.L. was supported by the European Union's H2020 R\&I Program-Marie Skłodowska-Curie GA 754510. M.D. was supported by the Spanish Association for Cancer Research (AECC). U.U. was supported by a BIST PhD fellowship. H.H. was supported by SAF2017-89109-P, AEI/FEDER, and UE. J.M.V. was supported by the Max Planck Society, the Deutsche Forschungsgemeinschaft (DFG) Priority Program SPP2202 Spatial Genome Architecture in Development and Disease (Project Ref. VA 1456/1), European Union H2020-Marie Sklodowska-Curie GA 643062-ZENCODEITN, and the Medical Research Council, UK. IRB Barcelona is a Severo Ochoa Center of Excellence (MINECO award SEV-2015-0505). We thank V.A. Raker for manuscript editing.

\section{AUTHOR CONTRIBUTIONS}

A.A. and S.A.B. designed the study. A.A. carried out most experiments. M.P.-G., M.D., A.C., and U.U.-U. assisted with experiments. N.P. performed the immunohistochemical analyses. A.V.K. assisted with mammary transplants. C.L. and Q.R. performed computational analyses. D.M. and H.H. generated scRNA-seq data and assisted with analyses. J.M.V. directed TE computational analysis. A.A. and S.A.B. wrote the manuscript.

\section{DECLARATION OF INTERESTS}

The authors declare no competing interests.

Received: April 24, 2020

Revised: January 18, 2021

Accepted: April 26, 2021

Published: May 18, 2021

\section{REFERENCES}

Avgustinova, A., and Benitah, S.A. (2016). Epigenetic control of adult stem cell function. Nat. Rev. Mol. Cell Biol. 17, 643-658.

Avgustinova, A., Symeonidi, A., Castellanos, A., Urdiroz-Urricelqui, U., SoléBoldo, L., Martín, M., Pérez-Rodríguez, I., Prats, N., Lehner, B., Supek, F., and Benitah, S.A. (2018). Loss of G9a preserves mutation patterns but increases chromatin accessibility, genomic instability and aggressiveness in skin tumours. Nat. Cell Biol. 20, 1400-1409.

Bankhead, P., Loughrey, M.B., Fernández, J.A., Dombrowski, Y., McArt, D.G., Dunne, P.D., McQuaid, S., Gray, R.T., Murray, L.J., Coleman, H.G., et al. (2017). QuPath: Open source software for digital pathology image analysis. Sci. Rep. 7, 16878 .

Benayoun, B.A., Pollina, E.A., Singh, P.P., Mahmoudi, S., Harel, I., Casey, K.M., Dulken, B.W., Kundaje, A., and Brunet, A. (2019). Remodeling of epigenome and transcriptome landscapes with aging in mice reveals widespread induction of inflammatory responses. Genome Res. 29, 697-709.

Bolger, A.M., Lohse, M., and Usadel, B. (2014). Trimmomatic: a flexible trimmer for Illumina sequence data. Bioinformatics 30, 2114-2120.

Buenrostro, J.D., Giresi, P.G., Zaba, L.C., Chang, H.Y., and Greenleaf, W.J. (2013). Transposition of native chromatin for fast and sensitive epigenomic profiling of open chromatin, DNA-binding proteins and nucleosome position. Nat. Methods 10, 1213-1218.

Burns, K.H. (2017). Transposable elements in cancer. Nat. Rev. Cancer 17, 415-424.

Bushnell, B., Rood, J., and Singer, E. (2017). BBMerge - Accurate paired shotgun read merging via overlap. PLoS ONE 12, e0185056.

Chuong, E.B., Elde, N.C., and Feschotte, C. (2017). Regulatory activities of transposable elements: from conflicts to benefits. Nat. Rev. Genet. 18, 71-86. Collado-Torres, L., Nellore, A., Frazee, A.C., Wilks, C., Love, M.I., Langmead, B., Irizarry, R.A., Leek, J.T., and Jaffe, A.E. (2017). Flexible expressed region analysis for RNA-seq with derfinder. Nucleic Acids Res. 45, e9.

Corrales, L., Woo, S.R., Williams, J.B., McWhirter, S.M., Dubensky, T.W., Jr., and Gajewski, T.F. (2016). Antagonism of the STING Pathway via Activation of the AIM2 Inflammasome by Intracellular DNA. J. Immunol. 196, 3191-3198.

De Cecco, M., Ito, T., Petrashen, A.P., Elias, A.E., Skvir, N.J., Criscione, S.W., Caligiana, A., Brocculi, G., Adney, E.M., Boeke, J.D., et al. (2019). L1 drives IFN in senescent cells and promotes age-associated inflammation. Nature $566,73-78$

Di Giacomo, M., Comazzetto, S., Sampath, S.C., Sampath, S.C., and O'Carroll, D. (2014). G9a co-suppresses LINE1 elements in spermatogonia. Epigenetics Chromatin 7, 24.

Dobin, A., Davis, C.A., Schlesinger, F., Drenkow, J., Zaleski, C., Jha, S., Batut, P., Chaisson, M., and Gingeras, T.R. (2013). STAR: ultrafast universal RNA-seq aligner. Bioinformatics 29, 15-21.

Ewald, A.J., Brenot, A., Duong, M., Chan, B.S., and Werb, Z. (2008). Collective epithelial migration and cell rearrangements drive mammary branching morphogenesis. Dev. Cell 14, 570-581

Feng, J., Liu, T., Qin, B., Zhang, Y., and Liu, X.S. (2012). Identifying ChIP-seq enrichment using MACS. Nat. Protoc. 7, 1728-1740.

Fernandes-Alnemri, T., Yu, J.W., Datta, P., Wu, J., and Alnemri, E.S. (2009). AIM2 activates the inflammasome and cell death in response to cytoplasmic DNA. Nature 458, 509-513. 


\section{$\infty$ CellPress}

\section{Cell Stem Cell

Godde, N.J., Sheridan, J.M., Smith, L.K., Pearson, H.B., Britt, K.L., Galea, R.C., Yates, L.L., Visvader, J.E., and Humbert, P.O. (2014). Scribble modulates the MAPK/Fra1 pathway to disrupt luminal and ductal integrity and suppress tumour formation in the mammary gland. PLoS Genet. 10, e1004323.

Gonzalez-Roca, E., Garcia-Albéniz, X., Rodriguez-Mulero, S., Gomis, R.R., Kornacker, K., and Auer, H. (2010). Accurate expression profiling of very small cell populations. PLoS ONE 5, e14418.

Gottardi, C.J., Arpin, M., Fanning, A.S., and Louvard, D. (1996). The junctionassociated protein, zonula occludens- 1 , localizes to the nucleus before the maturation and during the remodeling of cell-cell contacts. Proc. Natl. Acad. Sci. USA 93, 10779-10784.

Heinz, S., Benner, C., Spann, N., Bertolino, E., Lin, Y.C., Laslo, P., Cheng, J.X., Murre, C., Singh, H., and Glass, C.K. (2010). Simple combinations of lineagedetermining transcription factors prime cis-regulatory elements required for macrophage and B cell identities. Mol. Cell 38, 576-589.

Herquel, B., Ouararhni, K., Martianov, I., Le Gras, S., Ye, T., Keime, C., Lerouge, T., Jost, B., Cammas, F., Losson, R., and Davidson, I. (2013). Trim24-repressed VL30 retrotransposons regulate gene expression by producing noncoding RNA. Nat. Struct. Mol. Biol. 20, 339-346.

Hornung, V., Ablasser, A., Charrel-Dennis, M., Bauernfeind, F., Horvath, G., Caffrey, D.R., Latz, E., and Fitzgerald, K.A. (2009). AIM2 recognizes cytosolic dsDNA and forms a caspase-1-activating inflammasome with ASC. Nature $458,514-518$.

Huang, W., Sherman, B.T., and Lempicki, R.A. (2009). Systematic and integrative analysis of large gene lists using DAVID bioinformatics resources. Nat. Protoc. 4, 44-57.

Hutnick, L.K., Huang, X., Loo, T.C., Ma, Z., and Fan, G. (2010). Repression of retrotransposal elements in mouse embryonic stem cells is primarily mediated by a DNA methylation-independent mechanism. J. Biol. Chem. 285, 21082-21091.

Jin, Y., Tam, O.H., Paniagua, E., and Hammell, M. (2015). TEtranscripts: a package for including transposable elements in differential expression analysis of RNA-seq datasets. Bioinformatics 31, 3593-3599.

Johnson, W.E. (2019). Origins and evolutionary consequences of ancient endogenous retroviruses. Nat. Rev. Microbiol. 17, 355-370.

Jonkers, J., Meuwissen, R., van der Gulden, H., Peterse, H., van der Valk, M., and Berns, A. (2001). Synergistic tumor suppressor activity of BRCA2 and p53 in a conditional mouse model for breast cancer. Nat. Genet. 29, 418-425.

Jorgensen, I., Rayamajhi, M., and Miao, E.A. (2017). Programmed cell death as a defence against infection. Nat. Rev. Immunol. 17, 151-164.

Karimi, M.M., Goyal, P., Maksakova, I.A., Bilenky, M., Leung, D., Tang, J.X., Shinkai, Y., Mager, D.L., Jones, S., Hirst, M., and Lorincz, M.C. (2011). DNA methylation and SETDB1/H3K9me3 regulate predominantly distinct sets of genes, retroelements, and chimeric transcripts in mESCs. Cell Stem Cell 8, 676-687.

Kim, D., Pertea, G., Trapnell, C., Pimentel, H., Kelley, R., and Salzberg, S.L. (2013). TopHat2: accurate alignment of transcriptomes in the presence of insertions, deletions and gene fusions. Genome Biol. 14, R36.

Krause, S., Brock, A., and Ingber, D.E. (2013). Intraductal injection for localized drug delivery to the mouse mammary gland. J. Vis. Exp. (80).

Langmead, B., and Salzberg, S.L. (2012). Fast gapped-read alignment with Bowtie 2. Nat. Methods 9, 357-359.

Li, H., Handsaker, B., Wysoker, A., Fennell, T., Ruan, J., Homer, N., Marth, G., Abecasis, G., and Durbin, R.; 1000 Genome Project Data Processing Subgroup (2009). The Sequence Alignment/Map format and SAMtools. Bioinformatics 25, 2078-2079.

Liao, Y., Smyth, G.K., and Shi, W. (2014). featureCounts: an efficient general purpose program for assigning sequence reads to genomic features. Bioinformatics 30, 923-930.

Liu, S., Brind'Amour, J., Karimi, M.M., Shirane, K., Bogutz, A., Lefebvre, L., Sasaki, H., Shinkai, Y., and Lorincz, M.C. (2014). Setdb1 is required for germline development and silencing of H3K9me3-marked endogenous retroviruses in primordial germ cells. Genes Dev. 28, 2041-2055.
Liu, M., Thomas, S.L., DeWitt, A.K., Zhou, W., Madaj, Z.B., Ohtani, H., Baylin, S.B., Liang, G., and Jones, P.A. (2018). Dual Inhibition of DNA and Histone Methyltransferases Increases Viral Mimicry in Ovarian Cancer Cells. Cancer Res. 78, 5754-5766.

Love, M.I., Huber, W., and Anders, S. (2014). Moderated estimation of fold change and dispersion for RNA-seq data with DESeq2. Genome Biol. 15, 550. Maksakova, I.A., Thompson, P.J., Goyal, P., Jones, S.J., Singh, P.B., Karimi, M.M., and Lorincz, M.C. (2013). Distinct roles of KAP1, HP1 and G9a/GLP in silencing of the two-cell-specific retrotransposon MERVL in mouse ES cells. Epigenetics Chromatin 6, 15

Markopoulos, G., Noutsopoulos, D., Mantziou, S., Gerogiannis, D., Thrasyvoulou, S., Vartholomatos, G., Kolettas, E., and Tzavaras, T. (2016). Genomic analysis of mouse VL30 retrotransposons. Mob. DNA 7, 10.

Matsui, T., Leung, D., Miyashita, H., Maksakova, I.A., Miyachi, H., Kimura, H., Tachibana, M., Lorincz, M.C., and Shinkai, Y. (2010). Proviral silencing in embryonic stem cells requires the histone methyltransferase ESET. Nature 464, 927-931

Naik, S., Larsen, S.B., Gomez, N.C., Alaverdyan, K., Sendoel, A., Yuan, S., Polak, L., Kulukian, A., Chai, S., and Fuchs, E. (2017). Inflammatory memory sensitizes skin epithelial stem cells to tissue damage. Nature 550, 475-480.

Nakaya, Y., Lilue, J., Stavrou, S., Moran, E.A., and Ross, S.R. (2017). AIM2Like Receptors Positively and Negatively Regulate the Interferon Response Induced by Cytosolic DNA. mBio 8, e00944-17.

Page-McCaw, A., Ewald, A.J., and Werb, Z. (2007). Matrix metalloproteinases and the regulation of tissue remodelling. Nat. Rev. Mol. Cell Biol. 8, 221-233. Pal, B., Chen, Y., Vaillant, F., Jamieson, P., Gordon, L., Rios, A.C., Wilcox, S. Fu, N., Liu, K.H., Jackling, F.C., et al. (2017). Construction of developmental lineage relationships in the mouse mammary gland by single-cell RNA profiling. Nat. Commun. 8, 1627.

Ramírez, F., Ryan, D.P., Grüning, B., Bhardwaj, V., Kilpert, F., Richter, A.S., Heyne, S., Dündar, F., and Manke, T. (2016). deepTools2: a next generation web server for deep-sequencing data analysis. Nucleic Acids Res. 44 (W1), W160-5.

Reed, J.R., and Schwertfeger, K.L. (2010). Immune cell location and function during post-natal mammary gland development. J. Mammary Gland Biol. Neoplasia 15, 329-339.

Ritchie, M.E., Phipson, B., Wu, D., Hu, Y., Law, C.W., Shi, W., and Smyth, G.K. (2015). limma powers differential expression analyses for RNA-sequencing and microarray studies. Nucleic Acids Res. 43, e47.

Saini, S.K., Ørskov, A.D., Bjerregaard, A.M., Unnikrishnan, A., HolmbergThydén, S., Borch, A., Jensen, K.V., Anande, G., Bentzen, A.K., Marquard, A.M., et al. (2020). Human endogenous retroviruses form a reservoir of $T$ cell targets in hematological cancers. Nat. Commun. 11, 5660.

Schindelin, J., Arganda-Carreras, I., Frise, E., Kaynig, V., Longair, M., Pietzsch, T., Preibisch, S., Rueden, C., Saalfeld, S., Schmid, B., et al. (2012). Fiji: an open-source platform for biological-image analysis. Nat. Methods 9, 676-682. Shackleton, M., Vaillant, F., Simpson, K.J., Stingl, J., Smyth, G.K., AsselinLabat, M.L., Wu, L., Lindeman, G.J., and Visvader, J.E. (2006). Generation of a functional mammary gland from a single stem cell. Nature 439, 84-88.

Shi, J., Gao, W., and Shao, F. (2017). Pyroptosis: Gasdermin-Mediated Programmed Necrotic Cell Death. Trends Biochem. Sci. 42, 245-254.

Stark, R., and Brown, G. (2011). DiffBind: differential binding analysis of ChIPSeq peak data. https://bioconductor.org/packages/release/bioc/vignettes/ DiffBind/inst/doc/DiffBind.pdf.

Stuart, T., Butler, A., Hoffman, P., Hafemeister, C., Papalexi, E., Mauck, W.M., 3rd, Hao, Y., Stoeckius, M., Smibert, P., and Satija, R. (2019). Comprehensive Integration of Single-Cell Data. Cell 177, 1888-1902.e21.

Sun, P., Yuan, Y., Li, A., Li, B., and Dai, X. (2010). Cytokeratin expression during mouse embryonic and early postnatal mammary gland development. Histochem. Cell Biol. 133, 213-221.

Tachibana, M., Ueda, J., Fukuda, M., Takeda, N., Ohta, T., Iwanari, H., Sakihama, T., Kodama, T., Hamakubo, T., and Shinkai, Y. (2005). Histone methyltransferases G9a and GLP form heteromeric complexes and are both crucial for methylation of euchromatin at H3-K9. Genes Dev. 19, 815-826. 


\section{Cell Stem Cell}

\section{Article}

Tan, J., Buache, E., Alpy, F., Daguenet, E., Tomasetto, C.L., Ren, G.S., and Rio, M.C. (2014). Stromal matrix metalloproteinase-11 is involved in the mammary gland postnatal development. Oncogene 33, 4050-4059.

Tiwari, N., Meyer-Schaller, N., Arnold, P., Antoniadis, H., Pachkov, M., van Nimwegen, E., and Christofori, G. (2013). Klf4 is a transcriptional regulator of genes critical for EMT, including Jnk1 (Mapk8). PLoS ONE 8, e57329.

Unsworth, A., Anderson, R., Haynes, N., and Britt, K. (2016). OMIP-032: Two multi-color immunophenotyping panels for assessing the innate and adaptive immune cells in the mouse mammary gland. Cytometry A 89, 527-530.

Visvader, J.E., and Stingl, J. (2014). Mammary stem cells and the differentiation hierarchy: current status and perspectives. Genes Dev. 28, 1143-1158.

Waltman, L., and van Eck, N.J. (2013). A smart local moving algorithm for large-scale modularity-based community detection. Eur. Phys. J. B 86, 471.

Wiseman, B.S., Sternlicht, M.D., Lund, L.R., Alexander, C.M., Mott, J., Bissell, M.J., Soloway, P., Itohara, S., and Werb, Z. (2003). Site-specific inductive and inhibitory activities of MMP-2 and MMP-3 orchestrate mammary gland branching morphogenesis. J. Cell Biol. 162, 1123-1133.

Xu, C., and Su, Z. (2015). Identification of cell types from single-cell transcriptomes using a novel clustering method. Bioinformatics 31, 1974-1980.

Yu, Y.R., O'Koren, E.G., Hotten, D.F., Kan, M.J., Kopin, D., Nelson, E.R., Que, L., and Gunn, M.D. (2016). A Protocol for the Comprehensive Flow Cytometric Analysis of Immune Cells in Normal and Inflamed Murine Non-Lymphoid Tissues. PLoS ONE 11, e0150606.

Zevini, A., Olagnier, D., and Hiscott, J. (2017). Crosstalk between Cytoplasmic RIG-I and STING Sensing Pathways. Trends Immunol. 38, 194-205.

Zylicz, J.J., Dietmann, S., Günesdogan, U., Hackett, J.A., Cougot, D., Lee, C., and Surani, M.A. (2015). Chromatin dynamics and the role of G9a in gene regulation and enhancer silencing during early mouse development. eLife 4, 4. 


\section{STAR $\star$ METHODS}

\section{KEY RESOURCES TABLE}

\begin{tabular}{|c|c|c|}
\hline Reagent or Resource & Source & Identifier \\
\hline \multicolumn{3}{|l|}{ Antibodies } \\
\hline Rabbit polyclonal anti-collagen IV & abcam & Cat\#ab6586; RRID:AB_305584 \\
\hline Chicken polyclonal anti-cytokeratin 14 & Covance & Cat\#SIG-3476; RRID:AB_10718041 \\
\hline $\begin{array}{l}\text { Rat monoclonal anti-cytokeratin } \\
8 \text { (TROMA-I) }\end{array}$ & $\begin{array}{l}\text { Developmental Studies } \\
\text { Hybridoma Bank }\end{array}$ & Cat\#TROMA-I; RRID:AB_531826 \\
\hline Mouse monoclonal anti-dsDNA (3519 DNA) & abcam & Cat\#ab27156; RRID:AB_470907 \\
\hline Mouse monoclonal anti-dsDNA (AE-2) & Merck-Millipore & Cat\#MAB1293; RRID:AB_94097 \\
\hline Mouse monoclonal anti-E-cadherin (36) & BD Biosciences & Cat\#610182; RRID:AB_397581 \\
\hline Mouse monoclonal anti-ER $\alpha$ (1D5) & Dako & Cat\#M7047; RRID:AB_2101946 \\
\hline Rabbit polyclonal anti-G9a & Cell Signaling & Cat\#3306; RRID:AB_2097647 \\
\hline Mouse monoclonal anti-gH2ax (JBW301) & Merck-Millipore & Cat\#05-636; RRID:AB_309864 \\
\hline $\begin{array}{l}\text { Mouse monocolonal anti-H3K9me2 } \\
\text { (mAbcam 1220) }\end{array}$ & abcam & Cat\#ab1220; RRID:AB_449854 \\
\hline Mouse monoclonal anti-Ki67 (MM1) & Leica & Cat\#NCL-Ki67-MM1; RRID:AB_442101 \\
\hline Rat monoclonal anti-mouse CD16/32 (93) & BioLegend & Cat\#101320; RRID:AB_1574975 \\
\hline $\begin{array}{l}\text { Rabbit polyclonal anti-phospho-Ezrin } \\
\text { (Thr567)/Radixin (Thr564)/Moesin } \\
\text { (Thr558) (pERM) }\end{array}$ & Cell Signaling & Cat\#3141; RRID:AB_330232 \\
\hline $\begin{array}{l}\text { Rabbit polyclonal anti-zonula } \\
\text { occludens-1 (ZO-1) }\end{array}$ & Merck-Millipore & Cat\#AB2272; RRID:AB_10807434 \\
\hline Rat monoclonal anti-CD19 (6D5); PerCP & BioLegend & Cat\#115532; RRID:AB_2072926 \\
\hline $\begin{array}{l}\text { Rat monoclonal anti-Ly6C } \\
\text { (HK1.4); PerCP-Cy5.5 }\end{array}$ & BioLegend & Cat\#128011; RRID:AB_1659242 \\
\hline Rat monoclonal anti-CD24 (M1/69); PE-Cy7 & BioLegend & Cat\#101822; RRID:AB_756048 \\
\hline Rat monoclonal anti-CD8a (53-6.7); PE-Cy7 & BioLegend & Cat\#100722; RRID:AB_312761 \\
\hline $\begin{array}{l}\text { Armenian hamster monoclonal } \\
\text { anti-CD3e (1145-2C11); PE }\end{array}$ & BioLegend & Cat\#100308; RRID:AB_312673 \\
\hline Rat monoclonal anti-EpCAM (G8.8); PE & BioLegend & Cat\#118205; RRID:AB_1134176 \\
\hline $\begin{array}{l}\text { Rat monoclonal anti-CD62L } \\
\text { (MEL-14); BV711 }\end{array}$ & BioLegend & Cat\#104445; RRID:AB_2564215 \\
\hline $\begin{array}{l}\text { Rat monoclonal anti-IA/IE } \\
\text { (M5/114.15.2); BV650 }\end{array}$ & BioLegend & Cat\#107641; RRID:AB_2565975 \\
\hline Rat monoclonal anti-CD31 (390); BV605 & BioLegened & Cat\#102427; RRID:AB_2563982 \\
\hline Rat monoclonal anti-CD45 (30-F11); BV605 & BioLegend & Cat\#103140; RRID:AB_2562342 \\
\hline $\begin{array}{l}\text { Rat monoclonal anti-TER-119 } \\
\text { (TER-119); BV605 }\end{array}$ & BioLegend & Cat\#116239; RRID:AB_2562447 \\
\hline $\begin{array}{l}\text { Armenian hamster monoclonal } \\
\text { anti-CD11c (N418); BV421 }\end{array}$ & BioLegend & Cat\#117329; RRID:AB_10897814 \\
\hline $\begin{array}{l}\text { Rat monoclonal anti-CD11b } \\
\text { (M1/70); APC-Cy7 }\end{array}$ & BioLegend & Cat\#101225; RRID:AB_830641 \\
\hline $\begin{array}{l}\text { Rat monoclonal anti-EpCAM } \\
\text { (G8.8); APC-Cy7 }\end{array}$ & BioLegend & Cat\#118218; RRID:AB_2098648 \\
\hline $\begin{array}{l}\text { Armenian hamster monoclonal } \\
\text { anti-CD29 (HMb1-1); APC }\end{array}$ & BioLegend & Cat\#102216; RRID:AB_492833 \\
\hline Rat monoclonal anti-CD44 (IM7); APC & BioLegend & Cat\#103012; RRID:AB_312963 \\
\hline $\begin{array}{l}\text { Mouse monoclonal anti-CD64 } \\
\text { (X54-5/7.1); APC }\end{array}$ & BioLegend & Cat\#139305; RRID:AB_11219205 \\
\hline & & (Continued or \\
\hline
\end{tabular}




\section{Article}

\begin{tabular}{|c|c|c|}
\hline \multicolumn{3}{|l|}{ Continued } \\
\hline Reagent or Resource & Source & Identifier \\
\hline Rat monoclonal anti-CD4 (RM4-5); AF700 & BioLegend & Cat\#100536; RRID:AB_493701 \\
\hline Rat monoclonal anti-Ly6G (1A8) & BioLegend & Cat\#127621; RRID:AB_10640452 \\
\hline anti-FVS510 & BD Biosciences & Cat\#564406; RRID:AB_2869572 \\
\hline $\begin{array}{l}\text { Mouse monoclonal anti- } \alpha \text {-smooth } \\
\text { muscle actin (clone 1A4) }\end{array}$ & Sigma-Aldrich & Cat\#A5228; RRID:AB_262054 \\
\hline Goat anti-chicken IgY secondary Ab, AF647 & Life Technologies & Cat\#A21449; RRID:AB_1500594 \\
\hline Goat anti-mouse IgG secondary Ab, AF488 & Life Technologies & Cat\#A32723; RRID:AB_2633275 \\
\hline $\begin{array}{l}\text { Donkey anti-mouse IgG } \\
\text { secondary Ab, AF568 }\end{array}$ & Life Technologies & Cat\#A10037; RRID:AB_2534013 \\
\hline $\begin{array}{l}\text { Donkey anti-rabbit IgG } \\
\text { secondary Ab, AF568 }\end{array}$ & Life Technologies & Cat\#A21206; RRID:AB_2535792 \\
\hline $\begin{array}{l}\text { Donkey anti-rabbit IgG } \\
\text { secondary Ab, AF594 }\end{array}$ & Life Technologies & Cat\#A21207; RRID:AB_141637 \\
\hline Donkey anti-rat IgG secondary Ab, AF488 & Life Technologies & A21208; RRID:AB_141709 \\
\hline Rabbit monoclonal anti-p63 (EPR5701) & abcam & Cat\#ab124762; RRID:AB_10971840 \\
\hline \multicolumn{3}{|l|}{ Bacterial and virus strains } \\
\hline TRC1.5-pLKO.1-shRNA(Aim2) & Sigma-Aldrich & TRCN0000096107 \\
\hline pLKO.1-TRC control & Addgene & Cat\#10879; RRID:Addgene_10879 \\
\hline \multicolumn{3}{|l|}{ Chemicals, peptides, and recombinant proteins } \\
\hline Mitomycin-C & Sigma-Aldrich & Cat\#M4287 \\
\hline EpiCult-B Mouse Medium kit & StemCell Technologies & Cat\#05610 \\
\hline Hamś F12 & Life Technologies & Cat\#21765029 \\
\hline Adenine & Sigma-Aldrich & Cat\#A3159 \\
\hline Hydrocortisone & Sigma-Aldrich & Cat\#386698 \\
\hline Cholera enterotoxin & $\begin{array}{l}\text { Gentaur Molecular } \\
\text { Products BVBA }\end{array}$ & Cat\#100 \\
\hline Recombinant murine EGF & Peprotech & Cat\#315-09 \\
\hline Y-27632 & Sigma-Aldrich & Cat\#Y0503 \\
\hline Acetone & Sigma-Aldrich & Cat\#34859 \\
\hline Methanol & Sigma-Aldrich & Cat\#322415 \\
\hline Wrightś Giemsa & Sigma-Aldrich & Cat\#WG16 \\
\hline BD Matrigel Matrix & BD Biosciences & Cat\#354234 \\
\hline Collagenase/hyaluronidase & StemCell Technologies & Cat\#07912 \\
\hline RBC Lysis Buffer & eBioscience & Cat\#00-4333-57 \\
\hline Trypsin/EDTA & Life Technologies & Cat\#25200056 \\
\hline HBSS & Life Technologies & Cat\#14175053 \\
\hline Dispase II & Sigma-Aldrich & Cat\#D4693 \\
\hline DNase I & Sigma-Aldrich & Cat\#DN25 \\
\hline Collagenase I & Sigma-Aldrich & Cat\#C0130 \\
\hline EMEM $\left(\mathrm{Ca}^{2+}\right.$-free $)$ & Lonza & Cat\#BE06-174G \\
\hline PBS & Lonza & Cat\#H3BE17-516F \\
\hline $\begin{array}{l}\text { BD Matrigel Matrix (growth } \\
\text { factor-reduced) }\end{array}$ & BD Biosciences & Cat\#356230 \\
\hline Trypan blue & Sigma-Aldrich & Cat\#T8154 \\
\hline DAPI & Sigma-Aldrich & Cat\#32670 \\
\hline Absolute ethanol & VWR & Cat\#1.00983.2500 \\
\hline Chloroform & Sigma-Aldrich & Cat\#C2432 \\
\hline Glacial acetic acid & VWR & Cat\#1.01830.2500 \\
\hline Carmine & Sigma-Aldrich & Cat\#C1022 \\
\hline Aluminum potassium sulfate & Sigma-Aldrich & Cat\#A7167 \\
\hline
\end{tabular}




\begin{tabular}{|c|c|c|}
\hline \multicolumn{3}{|l|}{ Continued } \\
\hline Reagent or Resource & Source & Identifier \\
\hline Xylene & Sigma-Aldrich & Cat\#534056 \\
\hline CV Mount & Leica & Cat\#14046430011 \\
\hline Formaldehyde & Aname & Cat\#15710 \\
\hline BSA & Sigma-Aldrich & Cat\#A7906 \\
\hline Triton $\mathrm{X}$ & Sigma-Aldrich & Cat\#T8787 \\
\hline Vectashield & Vector laboratories & Cat\#H-1000 \\
\hline Formalin & Sigma-Aldrich & Cat\#HT501128 \\
\hline Donkey serum & Life technologies & Cat\#D9663 \\
\hline DAB & Dako & Cat\#K346711-2 \\
\hline Hematoxylin & Dako & Cat\#CS70030-2 \\
\hline Toluene-Free Mounting Medium & Dako & Cat\#CS70530-2 \\
\hline TRIzol & Thermo Scientific & Cat\#15596018 \\
\hline High-glucose DMEM & Life Technologies & Cat\#41965039 \\
\hline FBS & Life Technologies & Cat\#10270106 \\
\hline SYBR Select Master Mix & Life Technologies & Cat\#4472918 \\
\hline L-glutamine & Life Technologies & Cat\#25030024 \\
\hline Penicillin/Streptomycin & Life Technologies & Cat\#15140122 \\
\hline \multicolumn{3}{|l|}{ Critical commercial assays } \\
\hline TURBO DNA-free Kit & Thermo Fisher & Cat\#AM1907 \\
\hline Clariom S Mouse Array & Thermo Scientific & Cat\#901570 \\
\hline RevertAid First Strand cDNA Synthesis Kit & Thermo Scientific & Cat\#K1621 \\
\hline $\begin{array}{l}\text { TruSeq Stranded Total RNA Library Prep } \\
\text { Kit with Ribo-Zero Human/Mouse/Rat }\end{array}$ & Illumina & Cat\#RS-122-2201 \\
\hline FLICA 660 Caspase-1 Assay Kit & ImmunoChemistry & Cat\#9122 \\
\hline Lenti-X Concentrator & Takara Bio & Cat\#631231 \\
\hline Lenti-X p24 Rapid Titer Kit & Takara Bio & Cat\#632200 \\
\hline \multicolumn{3}{|l|}{ Deposited data } \\
\hline Raw and analyzed data & This paper & GEO: GSE149978 \\
\hline \multicolumn{3}{|l|}{ Experimental models: Cell lines } \\
\hline $\mathrm{NIH} / 3 \mathrm{~T} 3$ & ATCC & ATCC $®$ CRL-1658 \\
\hline HEK293T/17 & ATCC & ATCC $®$ CRL-11268 \\
\hline \multicolumn{3}{|l|}{ Experimental models: Organisms/strains } \\
\hline $\begin{array}{l}\text { Mouse: B6.129X1-Gt(ROSA) } \\
26 \text { or }^{\text {tm1(EYFP)Cos/J (Rosa26YFP) }}\end{array}$ & The Jackson Laboratory & Cat\#006148 \\
\hline Mouse: Crl:NU(Ico)-Foxn1 $1^{\text {nu }}$ (Swiss nude) & Charles River Laboratories & Cat\#620 \\
\hline $\begin{array}{l}\text { Mouse: B6N.Cg-Tg(KRT14-cre)1Amc/J } \\
\text { (K14-Cre) }\end{array}$ & The Jackson Laboratory & Cat\#018964 \\
\hline $\begin{array}{l}\text { Mouse: B6.Cg-Gt(ROSA) } \\
\text { 26Sor }{ }^{\text {tm9(CAG-tdTomato)Hze/J }} \\
\text { (Rosa26tdTomato) }\end{array}$ & The Jackson Laboratory & Cat\#007909 \\
\hline \multicolumn{3}{|l|}{ Software and algorithms } \\
\hline BowTie2 (version 2.3.3.1) & Langmead and Salzberg, 2012 & $\begin{array}{l}\text { http://bowtie-bio.sourceforge.net/ } \\
\text { bowtie2/index.shtml }\end{array}$ \\
\hline SAMtools (version 1.3.1) & Li et al., 2009 & http://samtools.sourceforge.net \\
\hline MACS2 (version 2.0.10) & Feng et al., 2012 & $\begin{array}{l}\text { https://github.com/macs3-project/ } \\
\text { MACS/wiki/Install-macs2 }\end{array}$ \\
\hline DiffBind (version 2.4.8) & Stark and Brown, 2011 & $\begin{array}{l}\text { https://www.bioconductor.org/packages/ } \\
\text { release/bioc/html/DiffBind.html }\end{array}$ \\
\hline HOMER (version 2015-03-22) & Heinz et al., 2010 & http://homer.ucsd.edu/homer/ \\
\hline Fiji (version 2.0.0-rc-68) & Schindelin et al., 2012 & https://imagej.net/Fiji \\
\hline
\end{tabular}




\section{Article}

\begin{tabular}{|c|c|c|}
\hline \multicolumn{3}{|l|}{ Continued } \\
\hline Reagent or Resource & Source & Identifier \\
\hline QuPath (version 0.1.2) & Bankhead et al., 2017 & https://qupath.github.io \\
\hline TopHat (version 2.1.1) & Kim et al., 2013 & $\begin{array}{l}\text { https://ccb.jhu.edu/software/ } \\
\text { tophat/index.shtml }\end{array}$ \\
\hline DESeq2 (version 1.26.0) & Love et al., 2014 & $\begin{array}{l}\text { https://bioconductor.org/packages/ } \\
\text { release/bioc/html/DESeq2.html }\end{array}$ \\
\hline DAVID (version 6.7) & Huang et al., 2009 & https://david.ncifcrf.gov \\
\hline limma (version 3.32.10) & Ritchie et al., 2015 & $\begin{array}{l}\text { https://www.bioconductor.org/packages/ } \\
\text { release/bioc/html/limma.html }\end{array}$ \\
\hline DERfinder (version 1.20.0) & Collado-Torres et al., 2017 & $\begin{array}{l}\text { https://bioconductor.org/packages/ } \\
\text { release/bioc/html/derfinder.html }\end{array}$ \\
\hline Seurat (version 3.0.1) & Stuart et al., 2019 & https://satijalab.org/seurat/ \\
\hline BBduk & Bushnell et al., 2017 & https://jgi.doe.gov/data-and-tools/bbtools \\
\hline STAR aligner (version STAR_2.5.2b) & Dobin et al., 2013 & https://github.com/alexdobin/STAR \\
\hline TEtranscript (version 2.0.3) & Jin et al., 2015 & $\begin{array}{l}\text { https://github.com/mhammell- } \\
\text { laboratory/TEtranscripts }\end{array}$ \\
\hline deeptools (version 3.3.1) & Ramírez et al., 2016 & $\begin{array}{l}\text { https://deeptools.readthedocs. } \\
\text { io/en/develop/ }\end{array}$ \\
\hline Prism (version 8.4.2) & GraphPad & https://www.graphpad.com \\
\hline FlowJo (version 10.4.2) & Treestar & https://www.flowjo.com \\
\hline Adobe Photoshop CS6 & Adobe & $\begin{array}{l}\text { https://www.adobe.com/products/ } \\
\text { photoshop.html }\end{array}$ \\
\hline Adobe Illustrator CS6 & Adobe & $\begin{array}{l}\text { https://www.adobe.com/products/ } \\
\text { illustrator.html }\end{array}$ \\
\hline Trimmomatic (version 0.36) & Bolger et al., 2014 & $\begin{array}{l}\text { http://www.usadellab.org/cms/? } \\
\text { page=trimmomatic }\end{array}$ \\
\hline \multicolumn{3}{|l|}{ Other } \\
\hline 34 G blunt end metal hub needle & Hamilton Company & Cat\#7803-05 \\
\hline Hamilton syringe $(50 \mu \mathrm{l})$ & Hamilton Company & Cat\#7637-01 \\
\hline
\end{tabular}

\section{RESOURCE AVAILABILITY}

\section{Lead contact}

Further information and requests for resources and reagents should be directed to and will be fulfilled by the lead contact, Salvador Aznar Benitah (salvador.aznar-benitah@irbbarcelona.org).

\section{Materials availability}

This study did not generate new unique reagents.

Data and code availability

RNA-seq (total and single-cell) and ATAC-seq data that support the findings of this study are deposited in the Gene Expression Omnibus (GEO) with accession number GEO: GSE149978. All other data supporting the findings of this study are available from the lead author on reasonable request. The software and algorithms for data analyses used in this study are all well-established from previous work and are referenced throughout the manuscript.

\section{EXPERIMENTAL MODEL AND SUBJECT DETAILS}

\section{Mouse strains}

Animal (Mus musculus) experiments were approved by the Ethical Committee for Animal Experimentation (CEEA) of the Scientific Park of Barcelona (PCB) and the Government of Catalunya and fully complied with their ethical regulations. All immunocompetent mice were maintained in a C57/BI6 genetic background. Rosa26YFP, Rosa26tdTomato, K14-Cre and athymic nude mice were obtained from Jackson Laboratories. G9a ${ }^{\text {fl/fl }}$ mice were kindly supplied by Dr. Yoichi Shinkai (Saitama University). Only female mice were used for studies. The developmental stage of the animals used is indicated in the figures and figure legends. Animals were housed in specific pathogen free (SPF) conditions. Allocation to WT or G9acKO groups was determined by the animal's genotype. Littermate controls (kept in the same cage) were used for all experiments. 


\section{$\infty$ CellPress}

\section{Cell Stem Cell

\section{Cell lines}

NIH 3T3 feeder cells and HEK293T cells (both from ATCC) were maintained in high-glucose DMEM (41965039, Life Technologies) containing 10\% FBS (10270106, Life Technologies), 2 mM L-glutamine (25030024, Life Technologies), and $100 \mu \mathrm{g} / \mathrm{ml}$ penicillin and streptomycin (15140122, Life Technologies) at $37^{\circ} \mathrm{C}$ and $5 \% \mathrm{CO}_{2}$. Prior to addition of mammary epithelial cells (also see "Clonogenic assays"), NIH 3T3 feeder cells were treated with $4 \mu \mathrm{g} / \mathrm{mL}$ mitomycin-C (M4287, Sigma-Aldrich) at $37^{\circ} \mathrm{C}$ for $2 \mathrm{~h}$ to arrest proliferation. Luminal cells were cultured using the EpiCult-B Mouse Medium kit (05610, StemCell Technologies) according to the manufacturer's instructions. Basal cells were maintained in FAD medium (three-parts DMEM [41965039], one-part Ham's F12 [21765029]; both from Life Technologies) containing 10\% FBS (10270106, Life Technologies), 2 mM L-glutamine (25030024, Life Technologies), $1.8 \times 10^{-4} \mathrm{M}$ adenine (A3159, Sigma-Aldrich), $0.5 \mathrm{~g} / \mathrm{ml}$ hydrocortisone (386698, Sigma-Aldrich), $5 \mu \mathrm{g} / \mathrm{ml}$ insulin, $1 \times 10^{-10}$ M cholera enterotoxin (100, Gentaur Molecular Products BVBA), $10 \mathrm{ng} / \mathrm{ml}$ EGF (315-09, Peprotech), and $10 \mu \mathrm{M}$ ROCKinhibitor Y-27632 (Y0503, Sigma-Aldrich). Primary cell cultures were maintained in normoxia conditions $\left(37^{\circ} \mathrm{C}, 10 \% \mathrm{CO}_{2}\right.$, and $\left.3 \% \mathrm{O}_{2}\right)$.

\section{METHOD DETAILS}

\section{Clonogenic assays}

For 2D colony formation assays, 500 luminal or basal cells were sorted directly into 6-well plates containing cell-type appropriate medium (see "Cell culture") and $1 \times 10^{5}$ mitomycin-C-treated NIH 3T3 feeder cells. After 7-10 days, colonies were fixed in icecold acetone:methanol (1:1 mixture; 34859 and 322415 respectively, both from Sigma-Aldrich) for 30 s and then air-dried. Colonies were stained in Wright's Giemsa (WG16, Sigma-Aldrich) for 3 min, washed, and air-dried. For 3D colony formation assays, FACSsorted cells were resuspended in 100\% Matrigel (354234, BD Biosciences), and gels were allowed to set for 30 min at $37^{\circ} \mathrm{C}$ before cell-appropriate culture medium was added. Spheroids were cultured for 10-14 days before fixation in 4\% paraformaldehyde. Quantification was carried out using Fiji software (version 2.0.0-rc-68) (Schindelin et al., 2012).

\section{Single-cell preparation}

Mammary epithelial cells were isolated using collagenase/hyaluronidase (07912, StemCell Technologies) digestion as detailed in the manufacturer's protocol. Briefly, mammary fat pads of 6- or 12-week-old female mice (as indicated in the figure legends) were digested at $37^{\circ} \mathrm{C}$ for $6 \mathrm{~h}$ under moderate agitation (750 rpm); red blood cells were then lysed using RBC Lysis Buffer (00-4333-57, eBioscience), and mammary epithelial fragments were further digested in $0.25 \%$ pre-warmed trypsin/EDTA (25200056, Life Technologies) for 2 min. Trypsin activity was neutralized with $2 \%$ FBS in HBSS (14175053, Life Technologies), followed by a final digest in prewarmed $5 \mathrm{mg} / \mathrm{ml}$ Dispase II (D4693, Sigma-Aldrich) containing 1 mg/ml DNase I (DN25, Sigma-Aldrich) for 2 min. Cell suspensions were filtered through $40-\mu \mathrm{m}$ cell strainers (SPL Life Sciences) and stained for flow cytometry.

For mammary-infiltrating immune-cell analysis, a shorter dissociation protocol was followed to maximize immune cell viability, as described previously (Unsworth et al., 2016). Briefly, $4^{\text {th }}$ inguinal mammary fat pads were collected, and lymph nodes were carefully removed; $4^{\text {th }}$ inguinal mammary fat pads were then mechanically dissociated using a Mcllwain Tissue Chopper (The Mickle Laboratory Engineering Co. LTD), and minced tissue was digested under gentle agitation (300 rpm) using $1.5 \mathrm{mg} / \mathrm{ml}$ collagenase I (C0130, Sigma-Aldrich) and $40 \mathrm{U} / \mathrm{ml}$ DNase I (DN25, Sigma-Aldrich) in calcium-free EMEM (BE06-174G, Lonza) for 60 min at $37^{\circ} \mathrm{C}$. Cells were pelleted, and red blood cells were lysed using RBC Lysis Buffer, washed twice in PBS (H3BE17-516F, Lonza), filtered through $40 \mu \mathrm{m}$ cell strainers (SPL Life Sciences) and stained for flow cytometry.

\section{Flow cytometry}

For flow cytometry analysis and sorting of mammary epithelial populations, single-cell suspensions from individual animals were resuspended to $1 \times 10^{7} \mathrm{cells} / \mathrm{ml}$ in PBS and labeled with EpCAM-PE, CD29-APC, and lineage-BV605 (CD31; CD45; TER119). Cells were re-suspended in $2 \mu \mathrm{g} / \mathrm{ml}$ DAPI (32670, Sigma-Aldrich) to exclude dead cells. Due to the presence of the ROSA26-YFP cassette in mice, mammary epithelial cells were positive for YFP. Luminal cells (EpCAMhigh/CD29low/lineage-) and basal cells (EpCAMlow/ CD29high/lineage-) were gated from YFP+ live cells and FACS-sorted using a BD FACSAria Fusion flow cytometer (BD Biosciences). For mammary transplants, mice containing ROSA26-tdTomato instead of ROSA26-YFP cassette were used, and total tdTomato+ mammary epithelium was sorted.

For immune analysis, an additional Fc-blocking step was performed prior to antibody incubation. The innate immune compartment was assayed and gated as previously described (Yu et al., 2016). The adaptive immune compartment was characterized following well-established protocols (Unsworth et al., 2016). Detailed information on all antibodies used is available in the Key resources table.

\section{Mammary transplants}

Mammary transplants were performed as previously described (Shackleton et al., 2006). FACS-sorted WT or G9acKO mammary epithelial cells (5000 Lin- cells per transplant) were resuspended in PBS containing 50\% growth factor-reduced Matrigel (356230, BD Biosciences) and $0.04 \%$ Trypan blue (T8154, Sigma-Aldrich) and injected in a $10 \mu$ l volume into the $4^{\text {th }}$ inguinal fat pad of 21 day-old female WT, G9acKO, or athymic nude mice. Prior clearing of endogenous mammary epithelium is indicated in the relevant figure legends. Recipient glands were removed for evaluation at 8 weeks post-transplant. Wholemounts of mammary outgrowths were stained as indicated below. An outgrowth was defined as an epithelial structure comprising ducts arising from a central point, with lobules and/or terminal end buds. 


\section{Cell Stem Cell} Article

\section{Lentiviral production}

Lentiviral particles were produced in HEK293T cells transfected with shCTR (pLKO.1, as a TRC control; a gift from David Root (Addgene plasmid \# 10879)), or shAim2 (TRCN0000096107, Sigma-Aldrich). Viral supernatants were concentrated using Lenti-X Concentrator (631231, Takara Bio) and viral particles were quantified using the Lenti-X p24 Rapid Titer Kit (632200, Takara Bio) according to the manufacturer's instructions.

Intraductal lentiviral injections

Intraductal injections of lentiviral particles were performed as previously described (Krause et al., 2013). Briefly, 4-week-old WT or G9acKO animals were anesthetized using isofluorane, and the hair in the nipple area was removed using over-the-counter hair removal cream. shCTR or shAim2 lentiviral particles $\left(3 \times 10^{6}\right)$ in $3 \mu \mathrm{L}$ PBS were injected into the nipple of the $4^{\text {th }}$ inguinal fat pad using a $34 \mathrm{G}$ blunt end metal hub needle (7803-05, Hamilton Company) affixed to a $50 \mu \mathrm{L}$ Hamilton syringe (7637-01, Hamilton Company). After 2 weeks, the $4^{\text {th }}$ inguinal fat pads were dissected and processed for downstream analyses.

\section{Caspase activity assay}

Caspase 1 activity was assessed using the fluorochrome-labeled inhibitors of caspases (FLICA) 660 Caspase-1 Assay Kit (9122, ImmunoChemistry) according to the manufacturer's instructions. Briefly, $1 \times 10^{6}$ mammary cells were incubated with $1 \times$ FLICA 660 reagent at $37^{\circ} \mathrm{C}$ for $1 \mathrm{~h}$. Cells were washed twice, incubated with primary antibodies (EpCAM-PE, CD31-BV605, CD45BV605, or TER-119-BV605), washed again, and re-suspended in $2 \mu \mathrm{g} / \mathrm{mL}$ DAPI (32670, Sigma-Aldrich) to be able to exclude dead cells. FLICA 660 levels within basal cells (EpCAMlow/Lin-) and luminal cells (EpCAMhigh/Lin-) were analyzed on a BD FACSAria Fusion flow cytometer (BD Biosciences).

\section{Mammary wholemounts}

For Carmine Alum-stained mammary wholemounts, $4^{\text {th }}$ inguinal mammary fat pads were dissected and fixed in Carnoy's fixative (60\% absolute ethanol [1.00983.2500, VWR], 30\% chloroform [C2432, Sigma-Aldrich], and 10\% glacial acetic acid [1018302500, VWR]) at room temperature overnight, rehydrated in sequential washes with decreasing concentrations of ethanol, and stained overnight in Carmine Alum Stain (0.2\% carmine [C1022, Sigma-Aldrich] and 0.5\% aluminum potassium sulfate [A7167, Sigma-Aldrich]). Wholemounts were once again dehydrated in ethanol and cleared in xylene (534056, Sigma-Aldrich) before mounting in CV Mount (14046430011, Leica Microsystems). Images were acquired on an Olympus MVX10 Macroscope and stitched together to create images covering the entire wholemount using MosaicJ plugin in Fiji. Quantification of branch and tip lengths was done using the ruler tool in Fiji. A branch was defined as the length of duct between two branch points. A tip was the length of branch from the most distal branch point to the tip of the mammary epithelium.

For fluorescent wholemounts, $4^{\text {th }}$ inguinal fat pads were dissected, fixed in $4 \%$ formaldehyde (15710, Aname) at room temperature for $2 \mathrm{~h}$, and blocked in PBS (H3BE17-516F, Lonza) containing 10\% BSA (A7906, Sigma-Aldrich), 5\% FBS (10270106, Life Technologies), and 1\% Triton X (T8787, Sigma-Aldrich) at room temperature for $3 \mathrm{~h}$. Wholemounts were incubated with primary antibodies diluted in blocking solution at room temperature overnight, washed three times in PBS, and incubated with secondary antibodies and DAPI diluted in blocking solution at room temperature for $3 \mathrm{~h}$. Mammary fat pads were washed and mounted using Vectashield $(\mathrm{H}-1000$, Vector laboratories) and imaged using a Leica TCS SP5 confocal microscope.

\section{Immunostaining}

Tissues were fixed in 10\% formalin (HT501128, Sigma-Aldrich) for $3 \mathrm{~h}$ at room temperature and embedded in paraffin; 4- $\mu \mathrm{m}$ sections were stained. For immunofluorescence, sections were blocked in 10\% donkey serum (D9663, Life Technologies) for $1 \mathrm{~h}$ at room temperature and stained with primary antibodies overnight at $4{ }^{\circ} \mathrm{C}$. Sections were washed three times in PBS before incubation with secondary antibodies for $1 \mathrm{~h}$ at room temperature. Slides were washed three times in PBS containing $2 \mu \mathrm{g} / \mathrm{mL}$ DAPI (D9542, Sigma-Aldrich) and mounted in Vectashield ( $\mathrm{H}-1000$, Vector laboratories). Immunofluorescence images were acquired using a Leica TCS SP5 or a Zeiss ELYRA PS. 1 confocal microscope. On occasion, the brightness of individual channels was adjusted in Fiji to aid visualization. Such modifications are indicated in the figure legends and were performed uniformly across all images presented for comparison. Raw data were used for quantification.

For immunohistochemistry, sections were stained with primary antibodies at room temperature for $2 \mathrm{~h}$, washed twice, and then incubated with secondary antibodies, followed by a 5-min incubation with DAB (K346711-2, Dako). Sections were counterstained with hematoxylin (CS70030-2, Dako) and mounted using Toluene-Free Mounting Medium (CS70530-2, Dako).

Stained immunohistochemistry sections were scanned using a high-resolution NanoZoomer 2.0 HT (Hamamatsu); images were quantified using the positive cell detection tool or the ruler tool of QuPath software (version 0.1.2) (Bankhead et al., 2017).

\section{ATAC sequencing}

Library preparation for ATAC (assay for transposase-accessible chromatin) sequencing used $5 \times 10^{4}$ luminal or basal cells (FACSsorted) from four WT or three G9caKO mice, as previously described (Buenrostro et al., 2013). Samples were sequenced on a HiSeq2500 sequencer (Illumina) using V4 chemistry, generating 50-bp paired-end reads. After adaptor-cleaning and quality correction using Trimmomatic (version 0.36) (Bolger et al., 2014), paired-end reads were aligned to the mm10 genome (UCSC) using BowTie 2 Aligner (version 2.3.3.1) (Langmead and Salzberg, 2012). Duplicate reads were removed using SAMtools (version 1.3.1) (Li et al., 


\section{$\infty$ CellPress}

\section{Cell Stem Cell

2009). Read alignment was offset as previously described (Buenrostro et al., 2013). To normalize for sequencing depth, the number of reads of all samples was downsampled to match the number of reads in the sample with the lowest coverage. Peaks were called using MACS2 (version 2.0.10) (Feng et al., 2012) and an FDR < 0.05. Differential peaks were determined using the DiffBind package (version 2.4.8) (Stark and Brown, 2011) in $\mathrm{R}$ (version 3.5.2) and an FDR $<0.05$. The distribution of the differential regions within genomic features was conducted with HOMER (version 2015-03-22) (Heinz et al., 2010).

Whole-genome expression analysis

For whole-genome expression profiling of mammary epithelial cells from 12 week old animals using total RNA sequencing, $2-5 \times 10^{5}$ luminal or basal cells were sorted and lysed in TRIzol (15596018, Thermo Scientific). RNA was extracted following the manufacturerś recommendations and treated with TURBO DNA-free Kit (AM1907, Thermo Fisher) prior to library preparation using TruSeq Stranded Total RNA Library Prep Kit with Ribo-Zero Human/Mouse/Rat Kit (RS-122-2201, Illumina). Samples were sequenced on a HiSeq2500 sequencer (Illumina) using V4 chemistry, generating 125-bp single reads. After adaptor-cleaning and quality correction using Trimmomatic (version 0.36), paired-end reads were aligned to the mm10 genome (UCSC) using TopHat Aligner (version 2.1.1) (Kim et al., 2013). Only uniquely aligning reads were used. For differentially expressed genes (DEGs), read count was performed using featureCounts (version 1.6.0) (Liao et al., 2014) and DEGs were called using DESeq2 (version 1.26.0) (Love et al., 2014), with adjusted $p$ value $<0.05$ and fold change $>1.5$.

Gene ontology (GO) analysis was performed using DAVID (version 6.7) (Huang et al., 2009) Genes from pyroptosis pathway were identified using the GO term GO:0070269 (Biological process, pyroptosis).

For whole-genome expression profiling of mammary epithelial cells from 6-week old animals, RNA was isolated and libraries prepared from 1000 cells as previously described (Gonzalez-Roca et al., 2010). cDNA was hybridized to the Clariom S Mouse Array (901570, Thermo Scientific) and scanned on a GeneAtlas Imaging station (Affymetrix). After RMA normalization and batch correction using the ComBat function of the SVA package in R, differentially expressed genes were determined using the limma package (version 3.32.10) (Ritchie et al., 2015) in $\mathrm{R}$ and an FDR $<0.05$.

For differentially expressed clusters (DECs), expressed regions along the whole genome were identified using the DERfinder package (version 1.20.0) in R (Collado-Torres et al., 2017), using a read cutoff of 5, maxClusterGap $=2000 \mathrm{~L}$, and maxRegionsGap $=0$. DECs identified using DESeq2 (FDR < 0.05) were filtered for intergenic regions. DECs were annotated using the annotateRegions function in R. TEs within DECs were considered differentially expressed.

\section{Real-time quantitative PCR (RT-qPCR)}

RNA was isolated as described above, followed by cDNA synthesis using the RevertAid First Strand cDNA Synthesis Kit (K1621, Thermo Scientific) according to the manufacturer's instructions. RT-qPCR analysis was performed using the SYBR Select Master Mix (4472918, Life Technologies) according the manufacturer's instructions. The reference gene Polr2a was used for normalization. Primer sequences are indicated in Table S7.

Single-cell RNA-sequencing (scRNA-seq)

Total mammary epithelial cells (YFP+ cells; see Figure S2A) were FACS-isolated from two WT and two G9acKO littermates. Cell concentration and viability were verified by counting with a TC20 Automated Cell Counter (Bio-Rad Laboratories, S.A). Cells were partitioned into Gel Bead-In-Emulsions in a Chromium Controller (10x Genomics) with a target cell recovery of 5000 cells. cDNA sequencing libraries were prepared using the Single-cell 3' mRNA kit (V3; $10 \times$ Genomics) following the manufacturer's instructions. Briefly, after GEM-RT clean-up, cDNA was amplified for 11 cycles, and cDNA quality control and quantification were performed on an Agilent Bioanalyzer High Sensitivity chip (Agilent Technologies). cDNA libraries were indexed by PCR using the PN-220103 Chromiumi7 Sample Index Plate. Size distribution and concentration of $3^{\prime}$ cDNA libraries were verified on an Agilent Bioanalyzer High Sensitivity chip (Agilent Technologies). Finally, cDNA libraries were sequenced on a HiSeq 4000 sequencer (Illumina) to obtain approximately 25,000-50,000 paired-end reads per cell, respectively.

\section{scRNA-seq analysis}

Alignment of scRNA.seq analyses was completed utilizing the $10 \times$ Genomics Cell Ranger pipeline (version 2.2.0). Each library was aligned to an indexed mm10 genome (USCS) using Cell Ranger Count and processed using the Seurat pipeline (version 3.0.1)(Stuart et al., 2019) in R (version 3.5.2). Only genes expressed in five or more cells were considered for the analysis. Cells were trimmed to those with $>200$ but $<4000$ unique genes, $<25 \%$ mitochondrially aligned reads, and $<50 \%$ of ribosomal reads. Doublets were removed using DoubletFinder and a doublet formation rate of $3 \%$. Gene expression levels were normalized and scaled using the NormalizeData and ScaleData functions in Seurat.

For each sample, the first 10 principal components (PCs) were selected for clustering. To cluster cells, a K-nearest neighbor (KNN) graph constructed on a Euclidean distance matrix in PCA space was calculated and then converted to a shared nearest neighbor (SNN) graph, in order to find highly interconnected communities of cells (Xu and Su, 2015). Cells were then clustered using the Louvain method to maximize modularity (Waltman and van Eck, 2013). Datasets were anchored and integrated using the Seurat v3 integrative analysis (FindlntegrationAnchors and IntegrateData functions) with a chosen dimensionality of 20.

Integrated data were then normalized and scaled as described above. Post anchoring, PCA was performed, and the first 30 PCs were used for UMAP dimensionality reduction, and subsequent clustering using the default Louvain implementation. 
The first 20 PCs were then used to construct an SNN matrix using the FindNeighbors function in Seurat with k.param set to 20. We then identified clusters using the FindClusters command with the resolution parameter set to 0.2 (or 0.1 in the case of basal subcluster identification). Marker genes per cluster were calculated using Seurat's FindAllMarkers( function and the "wilcox" test option. The full list of cluster markers is provided in Table S1. To find cluster-specific DEGs between conditions, a GLM-based method for singlecell differential expression analysis (MAST) within Seurat was used with default parameters. Cluster annotation was done using the AddModuleScore function using signatures genes for basal, luminal progenitor (LP), and mature luminal (ML) cell types obtained from previously published RNA-seq data (Pal et al., 2017).

Transposable element differential expression analysis

Before aligning, bulk RNA-seq sequencing reads were trimmed using BBduk software (Bushnell et al., 2017) with the following parameters: $k$ trim $=r, k=23$, mink $=11$, hdist $=1$, tbo, tpe. Trimmed reads were mapped using STAR aligner (version STAR_2.5.2b) (Dobin et al., 2013) with the parameters-sjdbOverhang 100,-winAnchorMultimapNmax 200 and-outFilterMultimapNmax 100 to the GRCm38 Mus musculus reference genome. Alignment files were sorted and indexed using samtools (version 1.5) (Li et al., 2009) . To count reads aligning to genes and TEs considering uniquely-mapping reads, TEtranscript software (version 2.0.3) (Jin et al., 2015) was used with the parameters -stranded reverse and -mode uniq. Gene annotations were downloaded from Gencode (Release M12, GRCm38.p5) and repeat annotations were downloaded from RepeatMasker (http://www.repeatmasker.org/). Repeats with the same ID were merged. Differential expression analysis was performed in R (version 3.4) using the DESeq2 package (version 1.18.1) (Love et al., 2014). TEs with adjusted $p$ value $\leq 0.01$ were considered significantly differentially expressed. The IfcShrink function from DESeq2 was used for visualization purposes.

Differential ATAC-seq peak enrichment analysis

Enrichment analysis of ATAC-seq peaks around differentially expressed genes and TEs was performed in R (version 3.4). For enrichment around differentially expressed genes, ATAC-seq peaks in WT and G9acKO were overlapped with differentially expressed genes (gene body $+1 \mathrm{~Kb}$ upstream of TSS) to construct a contingency table, and Fisher's exact test was used to determine significant association. For enrichment on TEs, presence or absence of ATAC-seq peak in WT or G9acKO was overlapped with all TE copies of each TE sub-family and tested for association with Fisher's exact test. After testing every TE subfamily, $p$ values were corrected for multiple testing using the p.adjust function with fdr method.

Heatmaps (Figures 4E and S4F) were generated using deepTools (version 3.3.1) (Ramírez et al., 2016) with option scale-regions to align the $5^{\prime}$ end and $3^{\prime}$ end of all the TE copies of the specific TE. Flanking region of $10 \mathrm{~Kb}$ upstream and downstream was included binned into bins. ATAC-seq open chromatin signal in the TE body was used to differentiate two clusters with -kmeans 2 option of plotheatmap function from deepTools.

\section{QUANTIFICATION AND STATISTICAL ANALYSIS}

Sample sizes, statistical tests, definitions of error bars, and $p$ values are indicated in figure legends. All statistical tests were twosided, and $p>0.05$ was considered as not significant (ns). Each experiment was repeated successfully at least twice with similar results. Statistical analyses were performed using Prism 8 (GraphPad) software. Flow cytometry data were analyzed using FlowJo 10 (Treestar). Adobe Photoshop CS6 and Adobe Illustrator CS6 were used for figure presentation. 